\title{
Optimization of non-coding regions for a non-modified mRNA COVID-19 vaccine
}

https://doi.org/10.1038/s41586-021-04231-6

Received: 13 August 2021

Accepted: 11 November 2021

Published online: 18 November 2021

\section{Open access}

Check for updates

\author{
Makda S. Gebre ${ }^{1,9}$, Susanne Rauch ${ }^{2,9 凶}$, Nicole Roth ${ }^{2}$, Jingyou Yu', Abishek Chandrashekar ${ }^{1}$, \\ Noe B. Mercado', Xuan He', Jinyan Liu', Katherine McMahan', Amanda Martinot ${ }^{3}$, \\ David R. Martinez ${ }^{4}$, Victoria Giffin', David Hope', Shivani Patel', Daniel Sellers', \\ Owen Sanborn', Julia Barrett', Xiaowen Liu ${ }^{5}$, Andrew C. Cole ${ }^{5}$, Laurent Pessaint ${ }^{6}$, \\ Daniel Valentin ${ }^{6}$, Zack Flinchbaugh ${ }^{6}$, Jake Yalley-Ogunro ${ }^{6}$, Jeanne Muench ${ }^{6}$, Renita Brown ${ }^{6}$, \\ Anthony Cook ${ }^{6}$, Elyse Teow ${ }^{6}$, Hanne Andersen ${ }^{6}$, Mark G. Lewis $^{6}$, Adrianus C. M. Boon ${ }^{7}$, \\ Ralph S. Baric ${ }^{4}$, Stefan O. Mueller ${ }^{2}$, Benjamin Petsch ${ }^{2,9}$ \& Dan H. Barouch ${ }^{1,8,9 凶}$
}

\begin{abstract}
The CVnCoV (CureVac) mRNA vaccine for severe acute respiratory syndrome coronavirus-2 (SARS-CoV-2) was recently evaluated in a phase $2 b / 3$ efficacy trial in humans ${ }^{1} . \mathrm{CV} 2 \mathrm{CoV}$ is a second-generation mRNA vaccine containing non-modified nucleosides but with optimized non-coding regions and enhanced antigen expression. Here we report the results of a head-to-head comparison of the immunogenicity and protective efficacy of $\mathrm{CVnCoV}$ and $\mathrm{CV} 2 \mathrm{CoV}$ in non-human primates. We immunized 18 cynomolgus macaques with two doses of $12 \mu \mathrm{g}$ lipid nanoparticle-formulated $\mathrm{CVnCoV}$ or $\mathrm{CV} 2 \mathrm{CoV}$ or with sham ( $n=6$ per group). Compared with $\mathrm{CVnCoV}, \mathrm{CV} 2 \mathrm{CoV}$ induced substantially higher titres of binding and neutralizing antibodies, memory $\mathrm{B}$ cell responses and $\mathrm{T}$ cell responses as well as more potent neutralizing antibody responses against SARS-CoV-2 variants, including the Delta variant. Moreover, $\mathrm{CV} 2 \mathrm{CoV}$ was found to be comparably immunogenic to the BNT162b2 (Pfizer) vaccine in macaques. Although CVnCoV provided partial protection against SARS-CoV-2 challenge, $\mathrm{CV} 2 \mathrm{CoV}$ afforded more robust protection with markedly lower viral loads in the upper and lower respiratory tracts. Binding and neutralizing antibody titres were correlated with protective efficacy. These data demonstrate that optimization of non-coding regions can greatly improve the immunogenicity and protective efficacy of a non-modified mRNASARS-CoV-2 vaccine in non-human primates.
\end{abstract}

Efficacy results in humans have recently been reported for the CVnCoV (CureVac) mRNA vaccine in the phase $2 \mathrm{~b} / 3$ HERALD trial in a population that included multiple viral variants. In this trial, the observed vaccine efficacy against symptomatic coronavirus disease 2019 (COVID-19) was approximately $48 \%$ and $53 \%$ in the overall study population and in a subgroup of participants $18-60$ years of age, respectively ${ }^{1}$. CV2CoV is a second-generation mRNA vaccine that incorporates modifications of non-coding regions that were selected by empiric screening for improved antigen expression ${ }^{2,3}$. Both $\mathrm{CVnCoV}$ and $\mathrm{CV} 2 \mathrm{CoV}$ are based on RNActive technology $y^{4-7}$ and consist of non-chemically modified sequence-engineered mRNA without pseudouridine ${ }^{6-12}$. Both vaccines encode the same full-length, pre-fusion stabilized severe acute respiratory syndrome coronavirus-2 (SARS-CoV-2) spike protein ${ }^{13,14}$ and are encapsulated in lipid nanoparticles (LNPs) with identical composition. $\mathrm{CV} 2 \mathrm{CoV}$ has been engineered with different non-coding regions flanking the open reading frame, which have previously been shown to improve transgene expression ${ }^{3}$ and protection against SARS-CoV-2 in
ACE2-transgenic mice ${ }^{2}$. Specifically, CV2CoV includes $5^{\prime}$ untranslated region (UTR) HSD17B4 and 3' UTR PSMB3 elements followed by a histone stem-loop motif and a poly(A) sequence (Fig. 1 and Methods). In the present study, we make a head-to-head comparison of the immunogenicity and protective efficacy of $\mathrm{CVnCoV}$ and $\mathrm{CV} 2 \mathrm{CoV}$ against SARS-CoV-2 challenge in non-human primates.

\section{Vaccine immunogenicity}

We immunized 18 cynomolgus macaques intramuscularly with $12 \mu \mathrm{g}$ $\mathrm{CVnCoV}, 12 \mu \mathrm{g} \mathrm{CV} 2 \mathrm{CoV}$ or sham vaccine (Fig. 1b). The animals were primed at week 0 and were boosted at week 4 . No clinical adverse effects were observed following vaccination. To assess innate immune responses, sera were isolated from all animals $24 \mathrm{~h}$ after the first vaccination to evaluate innate cytokine responses. $\mathrm{CV} 2 \mathrm{CoV}$ induced higher levels of IFN $\alpha 2 \mathrm{a}$, IP-10 and MIP-1 than CVnCoV $(P=0.0152, P=0.0152$ and $P=0.0411$, respectively; Extended Data Fig. 1$)$. 


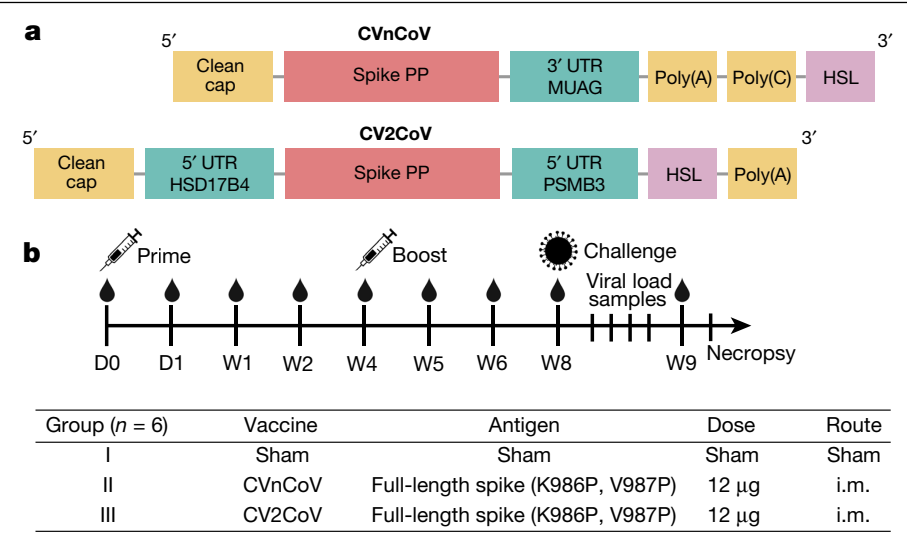

Fig. 1 | Vaccine design and study schema. a, Designs of the $\mathrm{CVnCoV}$ and $\mathrm{CV} 2 \mathrm{CoV}$ mRNA vaccine candidates. Both vaccines are based on CureVac's RNActive platform and encode SARS-CoV-2 spike protein with di-proline mutations. The vaccines differ in their unique non-coding regions, as shown. b, Non-human primate vaccine study schema. Cynomolgus macaques were immunized intramuscularly (i.m.) on day (D) 0 with $\mathrm{CVnCoV}(n=6)$ or $\mathrm{CV} 2 \mathrm{CoV}(n=6)$ mRNA vaccine or were designated as $\operatorname{sham}(n=6)$. The animals were boosted at week 4 and were challenged at week (W) 8. Samples were collected weekly after immunization and on days $0,1,2,4,7$ and 10 after challenge for immunological and virological assays. PP, K986P and V987P mutations; HSL, histone stem-loop.

Binding antibody responses were assessed by performing receptor-binding domain (RBD)-specific enzyme-linked immunosorbent assays (ELISAs) at multiple time points following immunization ${ }^{15,16}$. At week 2, binding antibody titres were detected only with $\mathrm{CV} 2 \mathrm{CoV}$ and not with $\mathrm{CVnCoV}$, with median values of 25 (range, 25-25) and 799 (range, 82-2,010) for CVnCoV and CV2CoV, respectively (Fig. 2a). One week after the week-4 boost, the antibody titres were increased in both groups, with medians of 48 (range, 75-710) and 28,407 (range, 2,714-86,541) for CVnCoV and CV2CoV, respectively (Fig. 2a). By week 8, the binding antibody titres had increased in the $\mathrm{CVnCoV}$ group but were still $>50$ times lower than those in the CV2CoV group $(P=0.0043)$, with median values of 214 (range, 47-1,238) and 14,827 (range, 2,133-37,079), respectively.

Neutralizing antibody responses were assessed by pseudovirus neutralization assay using the vaccine-matched SARS-CoV-2 wild-type (WT) WA1/2020 strain ${ }^{15-17}$. The neutralizing antibody titres followed a trend similar to that of the binding antibody titres (Fig. 2b). At week 2 , neutralizing antibodies were detected only with CV2CoV and not with $\mathrm{CVnCoV}$, with median values of 20 (range, 20-20) and 131 (range, 62-578) for CVnCoV and CV2CoV, respectively (Fig. 2b). One week after the week 4 boost, the neutralizing antibody titres were increased, with median values of 55 (range, 20-302) and 15,827 (range, 3,985-81,081) for $\mathrm{CVnCoV}$ and $\mathrm{CV} 2 \mathrm{CoV}$, respectively. By week 8 , the neutralizing antibody titres had increased in the $\mathrm{CVnCoV}$ group but were still $>20$ times lower than those in the CV2CoV group $(P=0.0022)$, with median values of 196 (range, 20-405) and 4,752 (range, 414-6,793), respectively.

At week 6, the median pseudovirus neutralizing antibody titres against the D614G, B.1.1.7 (Alpha) and B.1.351 (Beta) variants for CVnCoV were 121,101 and 189 , respectively, while they were $4,962,1,813$ and 755 for CV2CoV (Fig. 2c). The median pseudovirus neutralizing antibody titres against the C.37 (Lambda), B.1.617.1 (Kappa) and B.1.617.2 (Delta) variants for CVnCoV were 516, 158 and 36, respectively, while they were 1,195, 541 and 568 for CV2CoV (Extended Data Fig. 2). The pseudovirus neutralizing antibody titres induced by CV2CoV were higher than those induced by CVnCoV for the WT (WA1/2020), D614G, B.1.1.7 (Alpha), B.1.351 (Beta), C.37 (Lambda), B.1.617.1 (Kappa) and B.1.617.2 (Delta) strains $(P=0.0043,0.0087,0.0043,0.1320,0.026$, 0.0022 and 0.0043 , respectively). Taken together, these data show that $\mathrm{CV} 2 \mathrm{CoV}$ induces substantially higher pseudovirus neutralizing antibody titres against SARS-CoV-2 variants than CVnCoV.

Live-virus neutralizing antibody titre ${ }^{18}$ were largely consistent with those for the pseudovirus. The live-virus neutralizing antibody responses elicited by $\mathrm{CV} 2 \mathrm{CoV}$ were higher than those elicited by $\mathrm{CVn}$ CoV against the WA1/2020 and B.1.617.2 (Delta) strains $(P=0.0466$ and 0.0152, respectively), with similar trends for B.1.1.7 (Alpha) and B.1.351 (Beta) $(P=0.0628$ and 0.1450 , respectively) (Fig. $2 \mathrm{~d})$. a
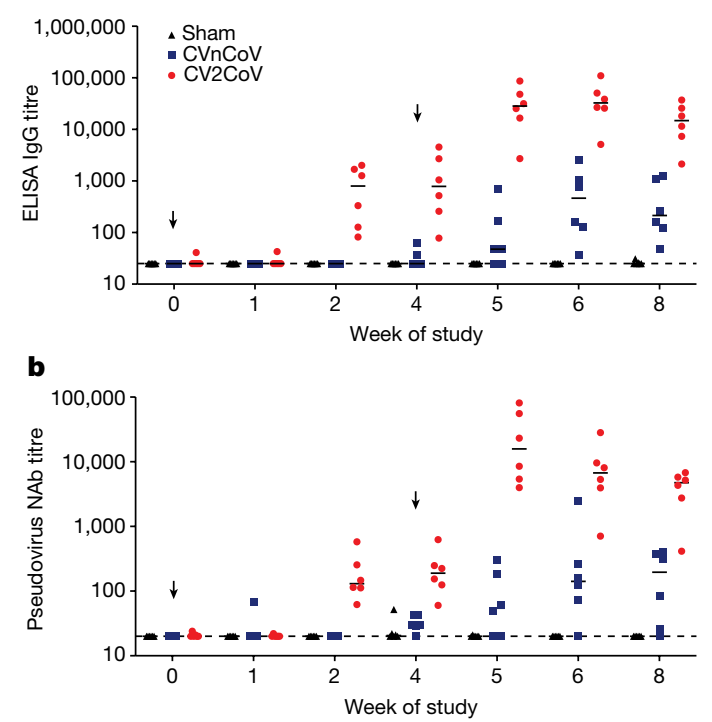

Fig. 2 |CV2CoV elicits high levels of binding and neutralizing antibody responses in macaques. Animals ( $n=6$ per group) were vaccinated twice with $12 \mu \mathrm{g}$ of $\mathrm{CVnCoV}$ or CV2CoV on day 0 and on day 28 or remained untreated as negative controls (sham). a, b, Titres of RBD-binding antibodies (a) and pseudovirus neutralizing antibodies (NAb) against the ancestral SARS-CoV-2 strain (b) were evaluated at different time points after the first (weeks $0,1,2$ and 4 ) and second (weeks 5, 6 and 8) vaccinations. c, d, Sera collected on day 42 (week 6)

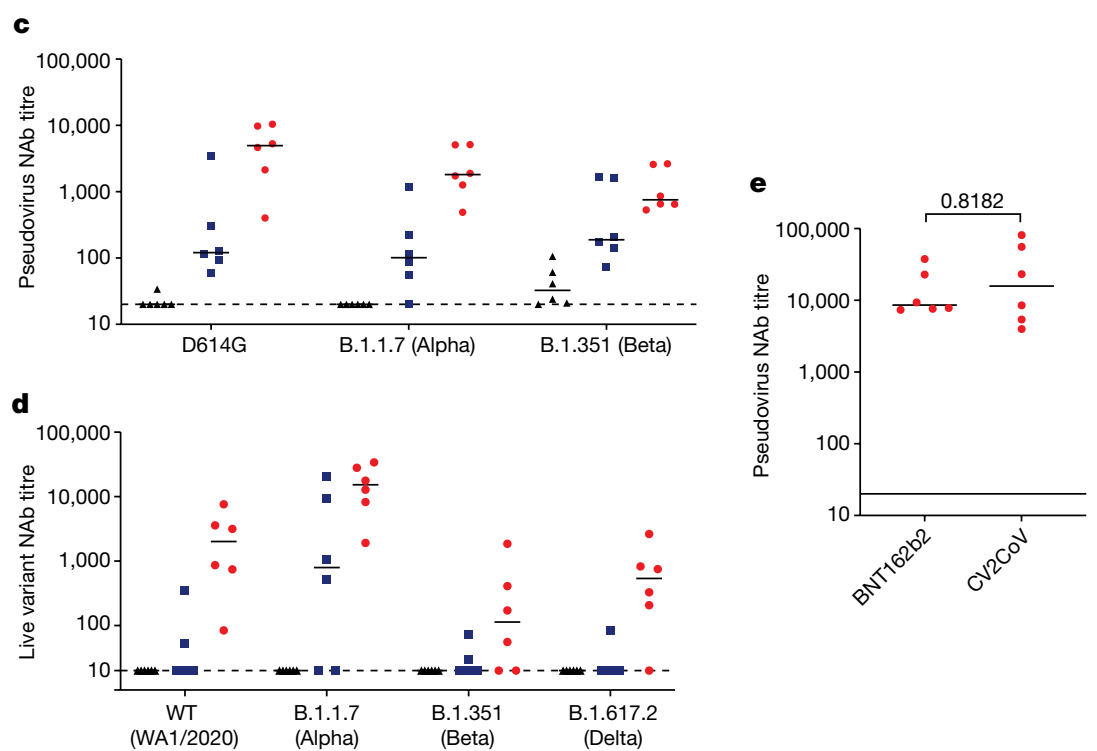

were analysed for pseudovirus (c) and live-virus (d) neutralizing antibody titres against virus with the D614G mutation and the B.1.1.7 (Alpha), B.1.351 (Beta) and B.1.617.2 (Delta) variants. e, Sera collected from non-human primates immunized with $12 \mu \mathrm{g}$ of CVnCoV or $30 \mu \mathrm{g}$ of BNT162b2 on day 35 (week 5) after boosting were analysed for pseudovirus neutralizing antibody titres against the ancestral WA/2020 (WT) strain. Each dot represents an individual animal and bars depict the median; the dashed line shows the limit of detection. 


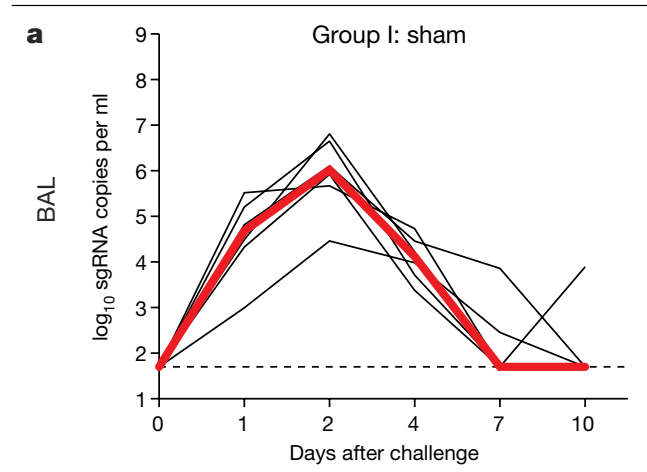

b

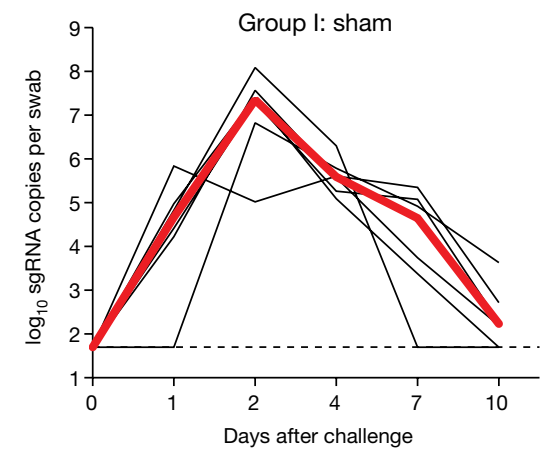

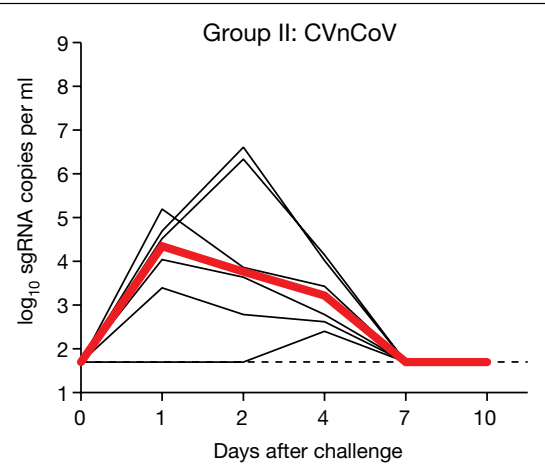

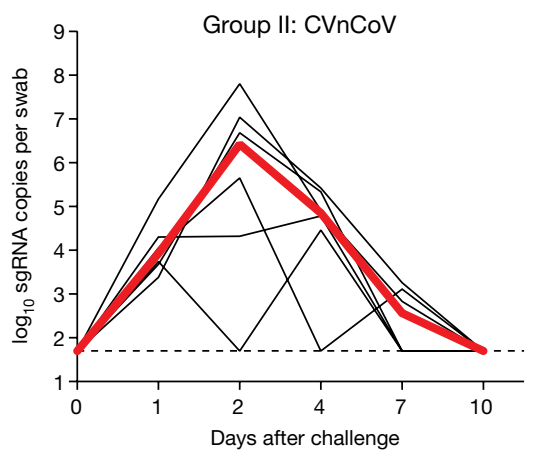

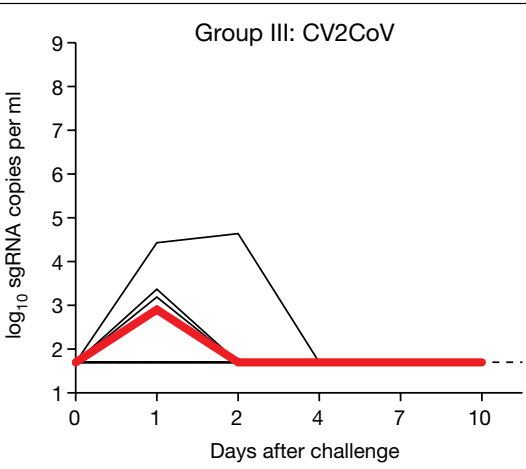

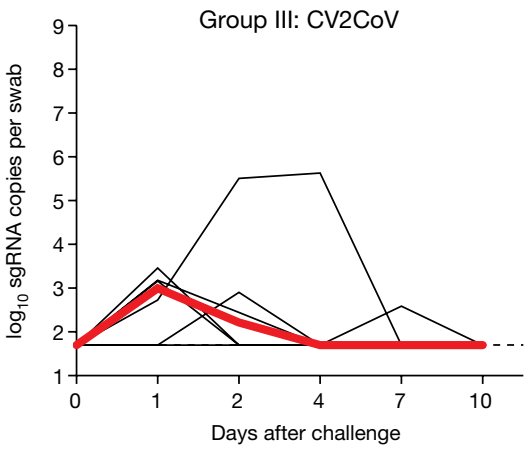

Fig. 3 | Protective efficacy of CV2CoV. Negative-control animals (sham) and animals ( $n=6$ per group) vaccinated on day 0 and day 28 with $12 \mu \mathrm{g}$ of $\mathrm{CVnCoV}$ or CV2CoV were challenged with $1.0 \times 10^{5} \mathrm{TCID}_{50}$ of SARS-CoV-2

(USA-WA1/2020) via the intranasal and intratracheal routes. a, b, BAL (a) and nasal swab (b) samples collected on days 1, 2, 4, 7 and 10 after challenge were analysed for levels of replicating virus by RT-PCR specific for sgRNA. Thin black lines represent individual animals and thick red lines depict the median; the dashed line shows the limit of detection.
We also compared the pseudovirus neutralizing antibody titres induced in macaques by two immunizations with $12 \mu \mathrm{g}$ of $\mathrm{CV} 2 \mathrm{CoV}$ to those induced by two immunizations with $30 \mu \mathrm{g}$ of the Pfizer BNT162b2 clinical vaccine obtained as leftover product from pharmacies. At peak immunity at week 5 , the neutralizing antibody responses induced by CV2CoV were comparable to those induced by BNT162b2 (Fig. 2e).

Most SARS-CoV-2 RBD-specific B cells reside within the memory $B$ cell pool ${ }^{19}$. We used flow cytometry to assess memory $B$ cell responses in the blood of non-human primates vaccinated with $\mathrm{CVnCoV}, \mathrm{CV} 2 \mathrm{CoV}$ or sham ${ }^{20}$. Higher numbers of RBD-specific and spike-specific memory $B$ cells were detected in the $\mathrm{CV} 2 \mathrm{CoV}$-vaccinated animals as compared with those vaccinated with $\mathrm{CVnCoV}$ at week $6(P=0.022$ and $P=0.0152$, respectively) (Extended Data Fig. 3a, b). T cell responses were assessed by interferon $\gamma$ (IFN $\gamma$ ) and interleukin (IL)-4 enzyme-linked immunosorbent spot (ELISPOT) assay using pooled spike peptides at week 6 . IFN $\gamma$ responses were detected in both groups but were higher in the CV2CoV group $(P=0.0065)$ (Extended Data Fig. 3c). IL-4 responses were not detectable, suggesting that $\mathrm{CVnCoV}$ and $\mathrm{CV} 2 \mathrm{CoV}$ induce $\mathrm{T}$ helper type 1-biased responses (Extended Data Fig. 3d).

\section{Protective efficacy}

All animals were challenged at week 8 with $1.0 \times 10^{5}$ median tissue culture infectious doses $\left(\right.$ TCID $\left._{50}\right)$ of the SARS-CoV-2 WA1/2020 strain via the intranasal and intratracheal routes. Viral loads were assessed in bronchoalveolar lavage (BAL) and nasal swab samples collected on days $1,2,4,7$ and 10 following challenge by quantitative PCR with reverse transcription (RT-PCR) specific for subgenomic RNA (sgRNA) ${ }^{21}$. The sgRNA levels in the BAL and nasal swab samples in the sham group peaked on day 2 and largely resolved by day 10 . The sham controls had peak medians of 6.02 (range, 4.62-6.81) $\log _{10}$-transformed sgRNA copies per $\mathrm{ml}$ in the BAL and 7.35 (range, 5.84-8.09) $\log _{10}$-transformed sgRNA copies per swab in the nasal swab samples on day 2 (Fig. 3). The CVnCoV-immunized animals showed peak medians of 4.92 (range,
2.40-6.61) $\log _{10}$-transformed sgRNA copies per $m \mathrm{~m}$ in the BAL and 6.42 (range, 4.46-7.81) $\log _{10}$-transformed sgRNA copies per swab in the nasal swab samples (Fig. 3). The CV2CoV-immunized animals exhibited peak medians of 2.90 (range, 1.70-4.64) $\log _{10}$-transformed sgRNA copies per $m l$ in the BAL and 3.17 (range, 2.59-5.63) $\log _{10}$-transformed sgRNA copies per swab in the nasal swab samples (Fig. 3), with resolution of sgRNA levels in the BAL samples by day 2 in most animals and by day 4 in all animals. Overall, $C V 2 \mathrm{CoV}$ resulted in significantly lower peak viral loads than $\mathrm{CVnCoV}$ in both the BAL $(P=0.0411)$ and nasal swab $(P=0.0087)$ samples (Fig. 4a, b).

We next evaluated the immune correlates of protection. The $\log _{10}$-transformed ELISA and neutralizing antibody titres at week 6 were inversely correlated with the peak $\log _{10}$-transformed sgRNA copies per $\mathrm{ml}$ in the BAL samples $(P=0.0008, R=-0.7148$ and $P=0.0015$, $R=-0.6912$, respectively, by two-sided Spearman rank-correlation test) (Fig. 4c, e) and with the peak sgRNA copies per nasal swab in the nasal swab samples $(P<0.0001, R=-0.8346$ and $P<0.0001, R=-0.8766$, respectively, by two-sided Spearman rank-correlation test) (Fig. $4 \mathrm{~d}, \mathrm{f}$ ). Consistent with prior observations from our laboratory and others ${ }^{15,16,22}$, these findings suggest that binding and neutralizing antibody titres are important correlates of protection for these SARS-CoV-2 vaccines in non-human primates. Similar correlates of protection were observed with viral loads assessed as area under the curve (Extended Data Fig. 4). Moreover, we assessed infectious virus titres by $\mathrm{TCID}_{50}$ assay on day 2 after challenge, which showed no detectable virus in five of six animals in the CV2CoV group (Extended Data Fig. 5).

Following challenge, we observed anamnestic binding and neutralizing antibody responses in all CVnCoV-vaccinated animals and in a subset of the CV2CoV-vaccinated animals ${ }^{16}$ (Extended Data Fig. 6). On day 10 after challenge, the animals were necropsied, and their lung tissues were evaluated by histopathology. Viral replication was largely resolved by day 10 in the animals vaccinated with $\mathrm{CVnCoV}$ and $\mathrm{CV} 2 \mathrm{CoV}$, and those with sham treatment had higher cumulative lung pathology scores ${ }^{19}$ (CVnCoV animals compared with sham controls, $P=0.0368 ; \mathrm{CV} 2 \mathrm{CoV}$ 

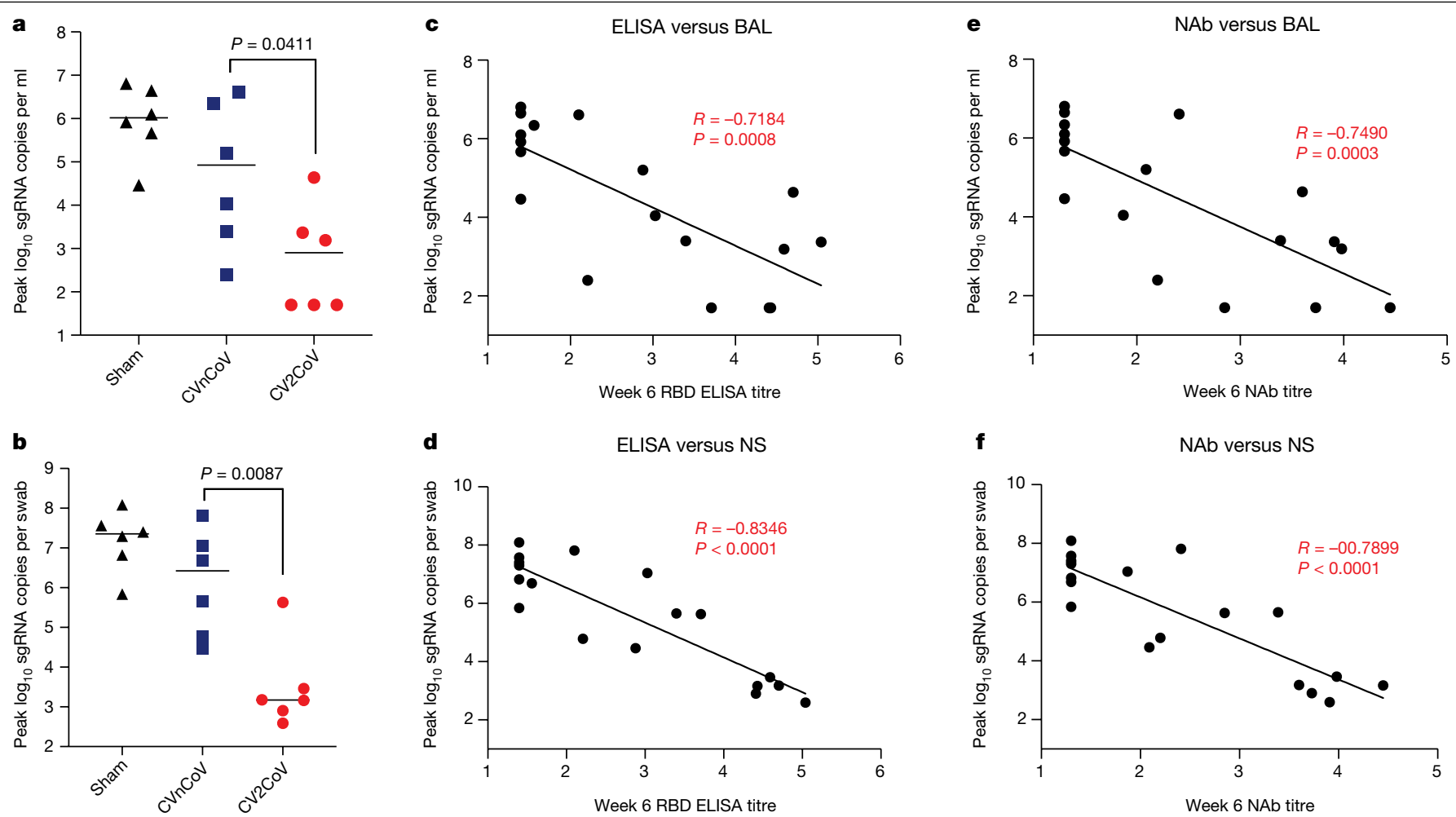

Fig. 4 | Titres of binding and neutralizing antibodies elicited following CVnCoV and CV2CoV vaccination ( $n=6$ per group) correlate with protection against SARS-CoV-2.a, b, Summary of peak viral loads following SARS-CoV-2 challenge in BAL and nasal swab (NS) samples. Animals were challenged with $1.0 \times 10^{5} \mathrm{TCID}_{50}$ of SARS-CoV-2 derived from strain
USA-WA1/2020 (NR-52281, BEI Resources). c-f, Antibody correlates of protection for binding antibodies $(\mathbf{c}, \mathbf{d})$ and neutralizing antibodies $(\mathbf{e}, \mathbf{f})$. Statistical analysis was performed using the two-tailed non-parametric Mann-Whitney test, and correlation was analysed by two-sided Spearman rank-correlation test. The bars indicate median values. animals compared with sham controls, $P=0.0022$ ) (Extended Data Fig. 7a). Animals in the sham group also had more lung lobes affected (Extended Data Fig. 7b) and more extensive lung lesions, with a greater proportion of lung lobes showing evidence of interstitial inflammation, alveolar inflammatory infiltrates and type II pneumocyte hyperplasia (Extended Data Fig. 7c-h). No significant eosinophilia was observed. The pathological lesions in vaccinated animals were similar to those observed for animals in the sham group (Extended Data Fig. 7i-l) but were overall fewer in number and more focal in distribution.

\section{Discussion}

CV2CoV elicited substantially greater humoral and cellular immune responses and provided significantly improved protective efficacy against SARS-CoV-2 challenge as compared with CVnCoV in macaques. These data suggest that optimization of non-coding elements of the mRNA backbone can substantially improve the immunogenicity and protective efficacy of mRNA vaccines. Both CVnCoV and CV2CoV contain only non-modified nucleosides with no pseudouridine or derivates, and $\mathrm{CV} 2 \mathrm{CoV}$ has previously been shown to lead to higher antigen expression than $\mathrm{CVnCoV}$ in cell culture ${ }^{3}$. The neutralizing antibody titres induced by $\mathrm{CV} 2 \mathrm{CoV}$ were comparable in macaques to those induced by the clinical BNT162b2 vaccine, which incorporates pseudouridine. These results suggest that strategies other than nucleoside modification can also markedly improve mRNA potency.

Previous studies with rodents and non-human primates have demonstrated protection by $\mathrm{CVnCoV}^{2,23,24}$. However, protection in macaques was primarily observed in the lower respiratory tract $\mathrm{t}^{23,24}$. In the present study, CVnCoV provided only modest viral load reductions in BAL and nasal swab samples compared with sham controls. In contrast to CVn$\mathrm{CoV}, \mathrm{CV} 2 \mathrm{CoV}$ induced $>10$-fold-higher neutralizing antibody responses against multiple viral variants and provided $>3 \log$ reductions in sgRNA copies per $\mathrm{ml}$ in BAL and $>4 \log$ reductions in sgRNA copies per swab in nasal swab samples compared with sham controls.

Previous mRNA vaccine clinical trials have demonstrated onset of protective efficacy after the first dose with improved protection after the boost immunization ${ }^{25,26}$. In the present study, the prime immunization with CV2CoV induced binding and neutralizing antibodies in all macaques by week 2 , and these responses had increased substantially by 1 week after the boost immunization. The neutralizing antibody titres induced by $\mathrm{CV} 2 \mathrm{CoV}$ in this study also appear to be similar to those reported for other mRNA vaccines in macaques ${ }^{27,28}$. Moreover, the neutralizing antibody titres induced by BNT162b2 in our study (Fig. 2e) were comparable to those reported for BNT162b2 in a prior study ${ }^{28}$.

As previously reported for other vaccines ${ }^{29-33}$, the neutralizing antibody titres against certain SARS-CoV-2 variants, such as the B.1.351 (Beta) and B.1.617.2 (Delta) variants, were lower than those against the parental strain WA1/2020. Although our challenge virus in this study was SARS-CoV-2 WA1/2020, the neutralizing antibody titres elicited by $\mathrm{CV} 2 \mathrm{CoV}$ to viral variants exceeded the values we previously reported as threshold titres for protection $(50-100)^{17,19,22}$. However, future studies will be required to directly assess the protective efficacy of $\mathrm{CV} 2 \mathrm{CoV}$ against SARS-CoV-2 variants of concern in non-human primates.

$\mathrm{CV} 2 \mathrm{CoV}$ induced both antigen-specific memory $\mathrm{B}$ cell responses and $T$ cell responses. Although the correlates of protection in this study were binding and neutralizing antibody titres ${ }^{34,35}$, it is likely that $C D 8^{+}$T cells contribute to viral clearance in tissues ${ }^{36,37}$. We previously reported that depletion of $\mathrm{CD}^{+} \mathrm{T}$ cells partially abrogated protective efficacy against SARS-CoV-2 re-challenge in convalescent macaques ${ }^{22}$. Memory B cells might contribute to the durability of antibody responses ${ }^{38,39}$; B cell germinal centre responses and the durability of protective efficacy following $\mathrm{CV} 2 \mathrm{CoV}$ vaccination remain to be determined. Moreover, 
although this study was not specifically designed as a safety study, it is worth noting that we did not observe any adverse effects following CVnCoV or CV2CoV vaccination, nor did we observe unexpected or enhanced pathology in the vaccinated animals at necropsy ${ }^{40}$.

In summary, our data show that optimization of non-coding regions in a SARS-CoV-2 mRNA vaccine can substantially improve its immunogenicity against multiple viral variants and can enhance its protective efficacy against SARS-CoV-2 challenge in macaques. The improved characteristics of $\mathrm{CV} 2 \mathrm{CoV}$ over those of $\mathrm{CVnCoV}$ might translate into increased efficacy in humans; accordingly, clinical trials of CV2CoV are planned.

\section{Online content}

Any methods, additional references, Nature Research reporting summaries, source data, extended data, supplementary information, acknowledgements, peer review information; details of author contributions and competing interests; and statements of data and code availability are available at https://doi.org/10.1038/s41586-021-04231-6.

1. Kremsner, P. G. et al. Efficacy and safety of the CVnCoV SARS-CoV-2 mRNA vaccine candidate: results from Herald, a phase $2 \mathrm{~b} / 3$, randomised, observer-blinded, placebo-controlled clinical trial in ten countries in Europe and Latin America. Lancet Infect. Dis. https://doi.org/10.1016/S1473-3099(21)00677-0 (2021).

2. Hoffmann, D. et al. CVnCoV and CV2CoV protect human ACE2 transgenic mice from ancestral B BavPat1 and emerging B.1.351 SARS-CoV-2. Nat. Commun. 12, 4048 (2021).

3. Roth, N. et al. CV2CoV, an enhanced mRNA-based SARS-CoV-2 vaccine candidate, supports higher protein expression and improved immunogenicity in rats. Preprint at https://doi.org/10.1101/2021.05.13.443734 (2021).

4. Hoerr, I., Obst, R., Rammensee, H. G. \& Jung, G. In vivo application of RNA leads to induction of specific cytotoxic T lymphocytes and antibodies. Eur. J. Immunol. 30 1-7 (2000).

5. Fotin-Mleczek, M. et al. Highly potent mRNA based cancer vaccines represent an attractive platform for combination therapies supporting an improved therapeutic effect. J. Gene Med. 14, 428-439 (2012).

6. Fotin-Mleczek, M. et al. Messenger RNA-based vaccines with dual activity induce balanced TLR-7 dependent adaptive immune responses and provide antitumor activity. J. Immunother. 34, 1-15 (2011).

7. Kubler, H. et al. Self-adjuvanted mRNA vaccination in advanced prostate cancer patients: a first-in-man phase I/Ila study. J. Immunother. Cancer 3, 26 (2015).

8. Lutz, J. et al. Unmodified mRNA in LNPs constitutes a competitive technology for prophylactic vaccines. NPJ Vaccines 2, 29 (2017).

9. Stitz, L. et al. A thermostable messenger RNA based vaccine against rabies. PLoS Negl. Trop. Dis. 11, e0006108 (2017).

10. Schnee, M. et al. An mRNA vaccine encoding rabies virus glycoprotein induces protection against lethal infection in mice and correlates of protection in adult and newborn pigs. PLoS Negl. Trop. Dis. 10, e0004746 (2016).

11. Petsch, B. et al. Protective efficacy of in vitro synthesized, specific mRNA vaccines against influenza A virus infection. Nat. Biotechnol. 30, 1210-1216 (2012).

12. Aldrich, C. et al. Proof-of-concept of a low-dose unmodified mRNA-based rabies vaccine formulated with lipid nanoparticles in human volunteers: a phase 1 trial. Vaccine $\mathbf{3 9}$, 1310-1318 (2021)

13. Pallesen, J. et al. Immunogenicity and structures of a rationally designed prefusion MERS-CoV spike antigen. Proc. Natl Acad. Sci. USA 114, E7348-E7357 (2017).

14. Kirchdoerfer, R. N. et al. Stabilized coronavirus spikes are resistant to conformational changes induced by receptor recognition or proteolysis. Sci. Rep. 8, 15701 (2018)

15. $\mathrm{Yu}$, J. et al. DNA vaccine protection against SARS-CoV-2 in rhesus macaques. Science 369, 806-811 (2020).

16. Mercado, N. B. et al. Single-shot Ad26 vaccine protects against SARS-CoV-2 in rhesus macaques. Nature 586, 583-588 (2020).
17. Chandrashekar, A. et al. SARS-CoV-2 infection protects against rechallenge in rhesus macaques. Science 369, 812-817 (2020).

18. Martinez, D. R. et al. Chimeric spike mRNA vaccines protect against Sarbecovirus challenge in mice. Science 373, 991-998 (2021).

19. He, X. et al. Low-dose Ad26.COV2.S protection against SARS-CoV-2 challenge in rhesus macaques. Cell https://doi.org/10.1016/j.cell.2021.05.040 (2021).

20. He, X. et al. Low-dose Ad26.COV2.S protection against SARS-CoV-2 challenge in rhesus macaques. Cell 184, 3467-3473 (2021).

21. Dagotto, G. et al. Comparison of subgenomic and total RNA in SARS-CoV-2 challenged rhesus macaques. J. Virol. https://doi.org/10.1128/JVI.02370-20 (2021).

22. McMahan, K. et al. Correlates of protection against SARS-CoV-2 in rhesus macaques. Nature 590, 630-634 (2021).

23. Rauch, S. et al. mRNA-based SARS-CoV-2 vaccine candidate $\mathrm{CVnCoV}$ induces high levels of virus-neutralising antibodies and mediates protection in rodents. NPJ Vaccines $\mathbf{6}$, 57 (2021).

24. Rauch, $\mathrm{S}$. et al. mRNA vaccine $\mathrm{CVnCoV}$ protects non-human primates from SARS-CoV-2 challenge infection. Preprint at https://doi.org/10.1101/2021.03.22.435960 (2020).

25. Polack, F. P. et al. Safety and efficacy of the BNT162b2 mRNA Covid-19 vaccine. N. Engl. J. Med. 383, 2603-2615 (2020).

26. Baden, L. R. et al. Efficacy and safety of the mRNA-1273 SARS-CoV-2 vaccine. N. Engl. J. Med. 384, 403-416 (2021).

27. Corbett, K. S. et al. Evaluation of the mRNA-1273 vaccine against SARS-CoV-2 in nonhuman primates. N. Engl. J. Med. 383, 1544-1555 (2020).

28. Vogel, A. B. et al. BNT162b vaccines protect rhesus macaques from SARS-CoV-2. Nature 592, 283-289 (2021)

29. Liu, C. et al. Reduced neutralization of SARS-CoV-2 B.1.617 by vaccine and convalescent serum. Cell https://doi.org/10.1016/j.cell.2021.06.020 (2021).

30. Haas, E. J. et al. Impact and effectiveness of mRNA BNT162b2 vaccine against SARS-CoV-2 infections and COVID-19 cases, hospitalisations, and deaths following a nationwide vaccination campaign in Israel: an observational study using national surveillance data. Lancet 397, 1819-1829 (2021).

31. Wu, K. et al. Serum neutralizing activity elicited by mRNA-1273 vaccine. N. Engl. J. Med. 384, 1468-1470 (2021).

32. Wibmer, C. K. et al. SARS-CoV-2 501Y.V2 escapes neutralization by South African COVID-19 donor plasma. Nat. Med. 27, 622-625 (2021).

33. Wall, E. C. et al. Neutralising antibody activity against SARS-CoV-2 VOCs B.1.617.2 and B.1.351 by BNT162b2 vaccination. Lancet 397, 2331-2333 (2021).

34. Philipp, M. \& Santibanez, G. Preference of respiratory phases to perform reaction time tasks. Act. Nerv. Super. 30, 153-155 (1988).

35. Feng, S. et al. Correlates of protection against symptomatic and asymptomatic SARS-CoV-2 infection. Nat. Med. 27, 2032-2040 (2021).

36. Lafon, E. et al. Potent SARS-CoV-2-specific T cell immunity and low anaphylatoxin levels correlate with mild disease progression in COVID-19 patients. Front. Immunol. 12, 684014 (2021).

37. Schmidt, M. E. \& Varga, S. M. The CD8 T cell response to respiratory virus infections. Front. Immunol. 9, 678 (2018)

38. Abayasingam, A. et al. Long-term persistence of $\mathrm{RBD}^{+}$memory $\mathrm{B}$ cells encoding neutralizing antibodies in SARS-CoV-2 infection. Cell Rep. Med. 2, 100228 (2021).

39. Dan, J. M. et al. Immunological memory to SARS-CoV-2 assessed for up to 8 months after infection. Science 371, https://doi.org/10.1126/science.abf4063 (2021).

40. Graham, B. S. Rapid COVID-19 vaccine development. Science 368, 945-946 (2020).

Publisher's note Springer Nature remains neutral with regard to jurisdictional claims in published maps and institutional affiliations.

Open Access This article is licensed under a Creative Commons Attribution 4.0 International License, which permits use, sharing, adaptation, distribution and reproduction in any medium or format, as long as you give appropriate credit to the original author(s) and the source, provide a link to the Creative Commons license, and indicate if changes were made. The images or other third party material in this article are included in the article's Creative Commons license, unless indicated otherwise in a credit line to the material. If material is not included in the article's Creative Commons license and your intended use is not permitted by statutory regulation or exceeds the permitted use, you will need to obtain permission directly from the copyright holder. To view a copy of this license, visit http://creativecommons.org/licenses/by/4.0/.

(c) The Author(s) 2021 


\section{Methods}

\section{mRNA vaccines}

The two mRNA vaccines, $\mathrm{CVnCoV}$ and $\mathrm{CV} 2 \mathrm{CoV}$, are based on CureVac's RNActive platform (claimed and described in, for example, patents WO2002098443 and WO2012019780) and include no chemically modified nucleosides. They are composed of a $5^{\prime}$ cap1 structure, a $\mathrm{G}+\mathrm{C}$-enriched open reading frame, a 3' UTR and a vector-encoded poly(A) stretch. CVnCoV contains a cleanCap (Trilink) and parts of the 3' UTR of the Homo sapiens alpha-haemoglobin gene as the 3' UTR, followed by a poly $(\mathrm{A})_{64}$ stretch, a poly $(\mathrm{C})_{30}$ stretch and a histone stem-loop ${ }^{22,23}$. CV2CoV has previously been described and contains a cleanCap followed by the $5^{\prime}$ UTR from the human hydroxysteroid 17-beta dehydrogenase 4 gene (HSD17B4) and a 3' UTR from the human proteasome $20 \mathrm{~S}$ subunit beta 3 gene (PSMB3) followed by a histone stem-loop and a poly $(\mathrm{A})_{100}$ stretch $^{3}$. The constructs were encapsulated in LNP by Acuitas Therapeutics (CV2CoV) or Polymun Scientific Immunbiologische Forschung (CVnCoV). LNPs are composed of ionizable amino lipid, phospholipid and cholesterol and PEGylated lipid; the compositions for $\mathrm{CVnCoV}$ and $\mathrm{CV} 2 \mathrm{CoV}$ are identical. Both mRNAs encode SARS-CoV-2 full-length spike protein containing stabilizing K986P and V987P mutations (NCBI reference sequence NC045512.2).

\section{Animals and study design}

Eighteen cynomolgus macaques of both sexes between the ages of 3 and 20 years were randomly assigned to three groups. The animals received either $\mathrm{CVnCoV}(n=6)$ or $\mathrm{CV} 2 \mathrm{CoV}(n=6)$ mRNA vaccine or were designated as sham controls $(n=6)$. The mRNA vaccines were administered intramuscularly at a $12-\mu \mathrm{g}$ dose in the left quadriceps on day 0 . Boost immunizations were similarly administered at week 4 . At week 8 , all animals were challenged with $1.0 \times 10^{5} \mathrm{TCID}_{50}$ of SARS-CoV-2 derived from USA-WA1/2020 (NR-52281, BEI Resources) ${ }^{17}$. The challenge virus was administered as $1 \mathrm{ml}$ by the intranasal route $(0.5 \mathrm{ml}$ in each naris) and $1 \mathrm{ml}$ by the intratracheal route. All animals were killed $10 \mathrm{~d}$ after challenge. Immunological and virological assays were performed with blinding. All animals were housed at Bioqual. All animal studies were conducted in compliance with all relevant local, state and federal regulations and were approved by the Bioqual Institutional Animal Care and Use Committee.

\section{Cytokine analyses}

The serum levels of 19 analytes that have been associated with immune response to viral infection were tested using the U-PLEX Viral Combo 1 (NHP) kit (K15069L-1) obtained from Meso Scale Discovery. The 19 analytes and their detection limits (LLODs) included G-CSF $\left(1.5 \mathrm{pg} \mathrm{ml}^{-1}\right)$, GM-CSF $\left(0.12 \mathrm{pg} \mathrm{ml}^{-1}\right)$, IFN $\alpha 2 \mathrm{a}\left(1.7 \mathrm{pg} \mathrm{ml}^{-1}\right)$, IFN $\gamma\left(1.7 \mathrm{pg} \mathrm{ml}^{-1}\right)$, IL-1RA $\left(1.7 \mathrm{pg} \mathrm{ml}^{-1}\right), \mathrm{IL}_{-1} 1\left(0.15 \mathrm{pg} \mathrm{ml}^{-1}\right), \mathrm{IL}-4\left(0.06 \mathrm{pg} \mathrm{ml}^{-1}\right), \mathrm{IL}-5\left(0.24 \mathrm{pg} \mathrm{ml}^{-1}\right)$, IL-6 $\left(0.33 \mathrm{pg} \mathrm{ml}^{-1}\right), \mathrm{IL}-7\left(1.5 \mathrm{pg} \mathrm{ml}^{-1}\right), \mathrm{IL}-8\left(0.15 \mathrm{pg} \mathrm{ml}^{-1}\right), \mathrm{IL}-9\left(0.14 \mathrm{pg} \mathrm{ml}^{-1}\right)$, IL-10 $\left(0.14 \mathrm{pg} \mathrm{ml}^{-1}\right)$, IL-12p70 $\left(0.54 \mathrm{pg} \mathrm{ml}^{-1}\right)$, IP-10 $\left(0.49 \mathrm{pg} \mathrm{ml}^{-1}\right)$, MCP-1 $\left(0.74 \mathrm{pg} \mathrm{ml}^{-1}\right), \operatorname{MIP}-1 \alpha\left(7.7 \mathrm{pg} \mathrm{ml}^{-1}\right)$, TNF $\left(0.54 \mathrm{pg} \mathrm{ml}^{-1}\right)$ and VEGF-A $\left(2.0 \mathrm{pg} \mathrm{ml}^{-1}\right)$. All serum samples were assayed in duplicate. The assay was performed by the Metabolism and Mitochondrial Research Core (Beth Israel Deaconess Medical Center, Boston, MA) following the manufacture's instructions. The assay plates were read by a MESO QuickPlex SQ 120 instrument, and the data were analysed using Discovery Workbench 4.0 software.

\section{ELISA}

RBD-specific binding antibodies were assessed by ELISA as described previously ${ }^{16,17}$. In brief, 96-well plates were coated with $1 \mu \mathrm{g} \mathrm{ml}^{-1}$ SARS-CoV-2 RBD protein (40592-VNAH, Sino Biological) in $1 \times$ DPBS and were incubated at $4{ }^{\circ} \mathrm{C}$ overnight. After incubation, the plates were washed once with wash buffer (0.05\% Tween-20 in $1 \times$ DPBS) and were blocked with $350 \mu \mathrm{l}$ casein block per well for $2-3 \mathrm{~h}$ at room temperature. After incubation, the block solution was discarded, and the plates were blotted dry. Serial dilutions of heat-inactivated serum diluted in casein block were added to the wells, and the plates were incubated for $1 \mathrm{~h}$ at room temperature. Next, the plates were washed three times and were then incubated for $1 \mathrm{~h}$ with a 1:1,000 dilution of anti-macaque IgG HRP (NIH NHP Reagent Program) at room temperature in the dark. The plates were then washed three more times, and $100 \mu \mathrm{l}$ of SeraCare KPL TMB SureBlue Start solution was added to each well; plate development was halted by the addition of $100 \mu \mathrm{l}$ of SeraCare KPL TMB Stop solution per well. The absorbance at $450 \mathrm{~nm}$ was recorded using a VersaMax or Omega microplate reader. The ELISA endpoint titres were defined as the highest reciprocal serum dilution that yielded an absorbance $>0.2$, and the $\log _{10}$ endpoint titres are reported. The immunological assays were performed with blinding.

\section{Pseudovirus neutralization assay}

SARS-CoV-2 pseudoviruses encoding a luciferase reporter gene were generated as described previously ${ }^{15}$. In brief, the packaging plasmid psPAX2 (AIDS Resource and Reagent Program), luciferase reporter plasmid pLenti-CMV Puro-Luc (Addgene) and spike protein-encoding pcDNA3.1-SARS CoV-2 S $\Delta$ CT plasmid of variants were co-transfected into HEK293T cells by Lipofectamine 2000 (ThermoFisher Scientific). Pseudoviruses of SARS-CoV-2 variants were generated by using the WA1/2020 strain (Wuhan/WIV04/2019; GISAID accession ID, EPI_ISL_402124), the strain with a D614G mutation, the B.1.1.7 variant (GISAID accession ID, EPI_ISL_601443), the B.1.351 variant (GISAID accession ID, EPI_ISL_712096), the C37 variant (GenBank ID, QRX62290), the B.1.671.1 variant (GISAID accession ID, EPI ISL_1384866) and the B.1.617.2 variant (GISAID accession ID, EPI_ISL_2020950). Supernatants containing the pseudotype viruses, which were purified by centrifugation and filtration with a $0.45-\mu \mathrm{m}$ filter, were collected $48 \mathrm{~h}$ after transfection. To determine the neutralization activity of the plasma or serum samples from the animals studied, HEK293T-hACE2 cells were seeded in 96-well tissue culture plates at a density of $1.75 \times 10^{4}$ cells per well overnight. Threefold serial dilutions of heat-inactivated serum or plasma samples were prepared and mixed with $50 \mu \mathrm{l}$ of pseudovirus. The mixture was incubated at $37^{\circ} \mathrm{C}$ for $1 \mathrm{~h}$ before being added to the HEK293T-hACE2 cells. The cells were lysed $48 \mathrm{~h}$ after infection in Steady-Glo Luciferase Assay buffer (Promega) according to the manufacturer's instructions. The SARS-CoV-2 neutralization titres were defined as the sample dilution at which a $50 \%$ reduction in relative light units (RLU) was observed relative to the average of the virus control wells.

\section{Live-virus neutralization assay}

Full-length SARS-CoV-2 WA1/2020, B.1.1.7, B.1.351 and B.1.617.2 viruses were designed to encode nanoluciferase (nLuc) and were recovered via reverse genetics ${ }^{18}$. One day before the assay, Vero E6 USAMRID cells were plated at 20,000 cells per well in clear-bottomed, black-walled plates. The cells were inspected to ensure confluency on the day of the assay. The serum samples were tested at a starting dilution of 1:20 and were serially diluted threefold for up to nine dilution spots. The serially diluted serum samples were mixed with diluted virus in an equal volume. The antibody-virus and virus-only mixtures were then incubated at $37^{\circ} \mathrm{C}$ with $5 \% \mathrm{CO}_{2}$ for $1 \mathrm{~h}$. After incubation, the serially diluted sera and virus-only controls were added in duplicate to the cells at 75 plaque-forming units at $37^{\circ} \mathrm{C}$ with $5 \%$ $\mathrm{CO}_{2}$. The cells were lysed $24 \mathrm{~h}$ later, and the luciferase activity was measured via Nano-Glo Luciferase Assay System (Promega) according to the manufacturer's specifications. The luminescence was measured by a Spectramax M3 plate reader (Molecular Devices). Virus neutralization titres were defined as the sample dilution at which a $50 \%$ reduction in $\mathrm{RLU}$ was observed relative to the average of the virus control wells. 


\section{B cell immunophenotyping}

Fresh peripheral blood mononuclear cells were stained with Aqua live/ dead dye (Invitrogen) for 20 min, washed with 2\% FBS in DPBS and suspended in 2\% FBS in DPBS with Fc Block (BD) for 10 min, followed by staining with monoclonal antibodies against CD45 (clone D058-1283, BUV805), CD3 (clone SP34.2, APC-Cy7), CD7 (clone M-T701, Alexa700), CD123 (clone 6H6, Alexa700), CD11c (clone 3.9, Alexa700), CD20 (clone 2H7, PE-Cy5), IgA (goat polyclonal antibodies, APC), IgG (clone G18-145, BUV737), IgM (clone G20-127, BUV396), IgD (goat polyclonal antibodies, PE), CD80 (clone L307.4, BV786), CD95 (clone DX2, BV711), CD27 (clone M-T271, BUV563), CD21 (clone B-ly4, BV605), CD14 (clone M5E2, BV570) and CD138 (clone DL-101, PE-CF594). The cells were also stained for SARS-CoV-2 antigens including biotinylated SARS-CoV-2 RBD protein (Sino Biological) and full-length SARS-CoV-2 spike protein (Sino Biological) labelled with FITC and DyLight 405 (DyLight 405 Conjugation Kit and FITC Conjugation Kit, Abcam) at $4{ }^{\circ} \mathrm{C}$ for 30 min. After staining, the cells were washed twice with 2\% FBS in DPBS, incubated with BV650 streptavidin (BD Pharmingen) for $10 \mathrm{~min}$ and then washed twice with 2\% FBS in DPBS. After staining, the cells were washed and fixed with $2 \%$ paraformaldehyde. All data were acquired on a BD FACSymphony flow cytometer. Subsequent analyses were performed using FlowJo software (Treestar, v.9.9.6). The immunological assays were performed with blinding.

\section{IFN $\gamma$ ELISPOT assay}

ELISPOT plates were coated with mouse anti-human IFN $\gamma$ monoclonal antibody (BD Pharmingen) at a concentration of $5 \mu$ g per well overnight at $4{ }^{\circ} \mathrm{C}$. The plates were washed with DPBS containing $0.25 \%$ Tween20 and were blocked with R10 medium (RPMI with 11\% FBS and 1.1\% penicillin-streptomycin) for $1 \mathrm{~h}$ at $37^{\circ} \mathrm{C}$. The S1 and S2 peptide pools (custom made,JPT Peptide Technologies) used in the assay contained peptides of 15 amino acids in length, overlapping by 11 amino acids, that spanned the protein sequence and reflect the $\mathrm{N}$-terminal and $\mathrm{C}$-terminal halves of the protein, respectively. The S1 and S2 peptide pools were prepared at a concentration of $2 \mu \mathrm{g}$ per well, and 200,000 cells per well were added. The peptides and cells were incubated for $18-24 \mathrm{~h}$ at $37^{\circ} \mathrm{C}$. All steps following this incubation were performed at room temperature. The plates were washed with ELISPOT wash buffer and were incubated for $2 \mathrm{~h}$ with $1 \mu \mathrm{g} \mathrm{ml}^{-1}$ rabbit polyclonal anti-human IFN $\gamma$ biotin obtained from U-Cytech. The plates were washed a second time and were then incubated for $2 \mathrm{~h}$ with $1 \mu \mathrm{g} \mathrm{ml}^{-1}$ streptavidin-alkaline phosphatase antibody obtained from Southern Biotech. The final wash was followed by the addition of nitro-blue tetrazolium chloride/5-bromo4-chloro $3^{\prime}$ indolyl phosphate $p$-toludine salt (NBT/BCIP chromagen) substrate solution (ThermoFisher Scientific) for $7 \mathrm{~min}$. The chromogen was discarded, and the plates were washed with water and were dried in a dim location for $24 \mathrm{~h}$. The plates were then scanned and counted using an ELISPOT analyser (Immunospot).

\section{IL-4 ELISPOT assay}

ELISPOT plates precoated with monoclonal antibody against IL-4 (Mabtech) were washed and blocked. The assay was then performed as described above except that the development time with NBT/BCIP chromagen substrate solution was $12 \mathrm{~min}$.

\section{Subgenomic RT-PCR assay}

SARS-CoV-2 Egene sgRNA was assessed by RT-PCR using primers and probes as previously described ${ }^{15,17}$. A standard was generated by first synthesizing a gene fragment of the subgenomic $E$ gene. The gene fragment was subsequently cloned into the pcDNA3.1+ expression plasmid using restriction site cloning (Integrated DNA Technologies). The insert was transcribed in vitro to RNA using the AmpliCap-Max T7 High Yield Message Maker kit (CellScript). Log dilutions of the standard were prepared for RT-PCR assays, ranging from $1 \times 10^{10}$ copies to $1 \times 10^{-1}$ copies. The viral loads were quantified from BAL fluid and nasal swab samples. RNA extraction was performed on a QIAcube HT using the IndiSpin QIAcube HT Pathogen kit according to the manufacturer's specifications (Qiagen). The standard dilutions and extracted RNA samples were reverse-transcribed using SuperScript VILO Master Mix (Invitrogen) following the cycling conditions described by the manufacturer. A Taqman custom gene expression assay (ThermoFisher Scientific) was designed using the sequences targeting the $E$ gene sgRNA. The sequences for the custom assay were as follows: forward primer, sgLeadCoV2.Fwd: 5'-CGATCTCTTGTAGATCTGTTCTC-3'; E Sarbeco_R:5'-ATATTGCAGCAGTACGCACACA-3'; E_Sarbeco_P1 (probe): 5'-VIC-ACACTAGCCATCCTTACTGCGCTTCG-MGBNFQ-3'. Reactions were carried out in duplicate for samples and standards on QuantStudio 6 and 7 Flex Real-Time PCR systems (Applied Biosystems) with the following thermal cycling conditions: initial denaturation at $95^{\circ} \mathrm{C}$ for $20 \mathrm{~s}$ followed by 45 cycles of $95^{\circ} \mathrm{C}$ for $1 \mathrm{~s}$ and $60^{\circ} \mathrm{C}$ for $20 \mathrm{~s}$. Standard curves were used to calculate the sgRNA copies per millilitre or per swab. The quantitative assay sensitivity was determined as 50 copies per millilitre or per swab.

\section{TCID $_{50}$ assay}

Vero TMPRSS2 cells (obtained from A. Creanga, NIH) were plated at 25,000 cells per well in DMEM with $10 \% \mathrm{FBS}$ and gentamicin, and the cultures were incubated at $37^{\circ} \mathrm{C}, 5.0 \% \mathrm{CO}_{2}$. Medium was aspirated and replaced with $180 \mu$ l of DMEM with $2 \%$ FBS and gentamicin. Serial dilution of samples as well as positive (virus stock of known infectious titre) and negative (medium only) controls were included in each assay. The plates were incubated at $37^{\circ} \mathrm{C}, 5.0 \% \mathrm{CO}_{2}$, for $4 \mathrm{~d}$, and the cell monolayers were visually inspected for cytopathic effects. TCID $_{50}$ was calculated using the Read-Muench formula.

\section{Histopathology}

At the time of fixation, lungs were suffused with $10 \%$ formalin to expand the alveoli. All tissues were fixed in $10 \%$ formalin and block-sectioned at $5 \mu \mathrm{m}$. The slides were baked for $30-60 \mathrm{~min}$ at $65^{\circ} \mathrm{C}$, deparaffinized in xylene, rehydrated through a series of graded ethanol to distilled water and then stained with haematoxylin and eosin. Blinded histopathological evaluation was performed by a board-certified veterinary pathologist (A.J.M.).

\section{Statistical analyses}

Statistical analyses were performed using GraphPad Prism (version 9.0) software (GraphPad Software), and comparisons between groups were performed using a two-tailed non-parametric Mann-Whitney $U$ test. $P$ values of less than 0.05 were considered as significant. Correlations were assessed by applying two-sided Spearman rank-correlation tests.

\section{Reporting summary}

Further information on research design is available in the Nature Research Reporting Summary linked to this paper.

\section{Data availability}

All data are available in the manuscript and its Supplementary Information. Source data are provided with this paper.

Acknowledgements We thank S. Gardner, G. Kennedy and R. Edmonston for their assistance and D. Maione and M.-T. Martin for their analysis of the manuscript. This work was supported by CureVac AG and the German Federal Ministry of Education and Research (BMBF; 01KI20703), the National Institutes of Health (CA260476), the Massachusetts Consortium on Pathogen Readiness and the Ragon Institute of MGH, MIT and Harvard. Development of CV2CoV is conducted in a collaboration of CureVac AG and GSK.

Author contributions S.R., B.P., S.O.M., N.R. and D.H.B. designed the study. M.S.G., J.Y., A. Chandrashekar, N.B.M., X.H., J.L., K.M., A.M., D.R.M., R.S.B., A.C.M.B., V.G., D.H., S.P., D.S., O.S and J.B. performed immunological and virological assays. X.L. and A.C.C. performed cytokine 
analysis. L.P., D.V., Z.F., J.Y.-O., J.M., R.B., A. Cook, E.T., H.A. and M.G.L. led the clinical care of the animals. M.S.G. and D.H.B. wrote the manuscript with all co-authors.

Competing interests S.R., B.P., N.R. and S.O.M. are employees of CureVac AG, Tübingen,

Germany, a publicly listed company developing mRNA-based vaccines and

immunotherapeutics. Authors may hold shares in the company. S.R., B.P. and N.R. are inventors

on several patents on mRNA vaccination and use thereof. The other authors declare no

competing interests.
Additional information

Supplementary information The online version contains supplementary material available at https://doi.org/10.1038/s41586-021-04231-6.

Correspondence and requests for materials should be addressed to Susanne Rauch or Dan H. Barouch.

Peer review information Nature thanks Wolfgang Baumgärtner and the other, anonymous, reviewer(s) for their contribution to the peer review of this work.

Reprints and permissions information is available at http://www.nature.com/reprints. 


\section{Article}

a

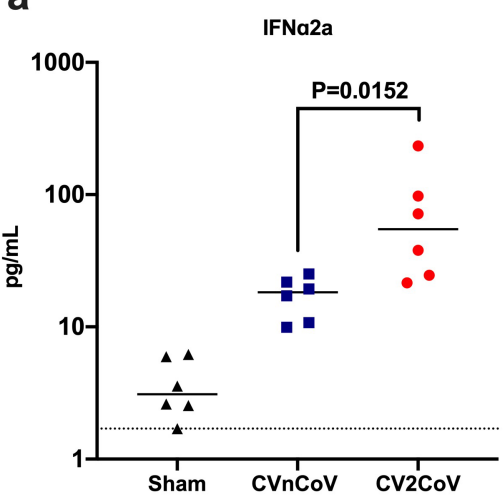

d

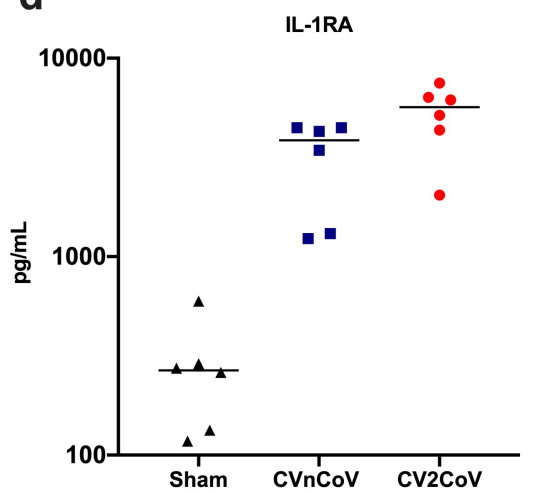

g

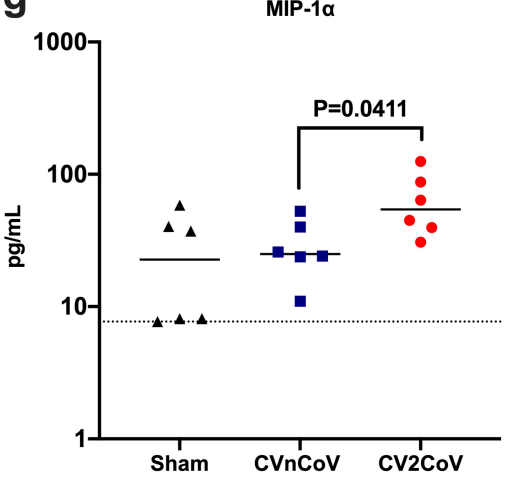

b

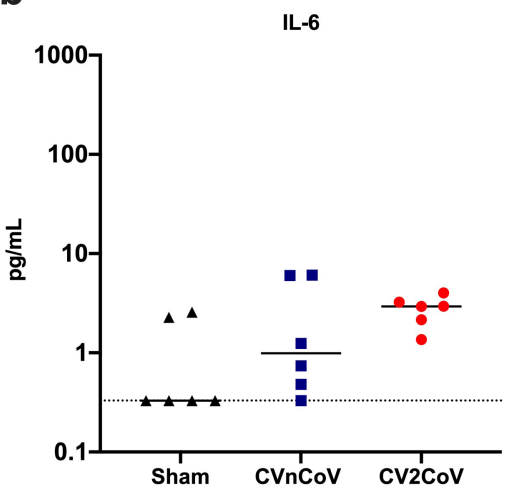

e

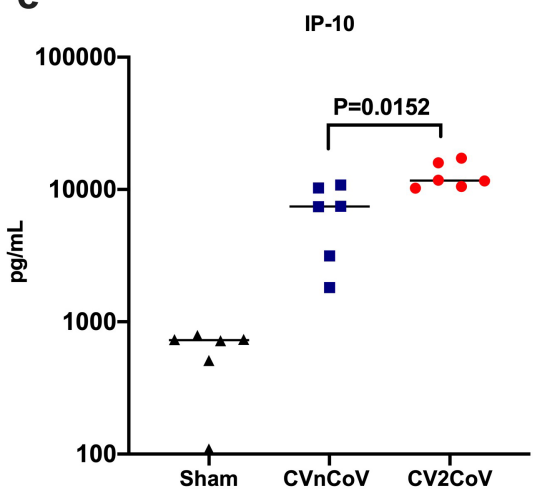

h

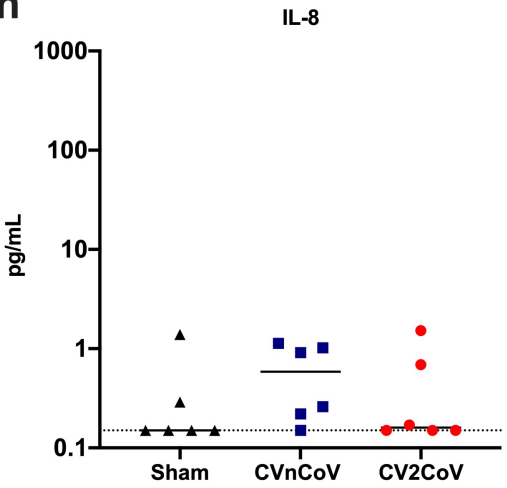

C

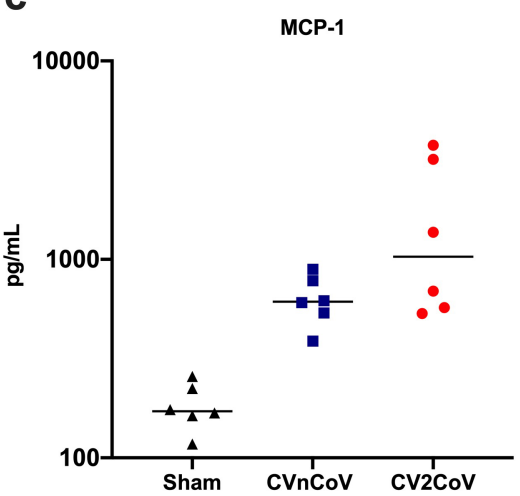

f

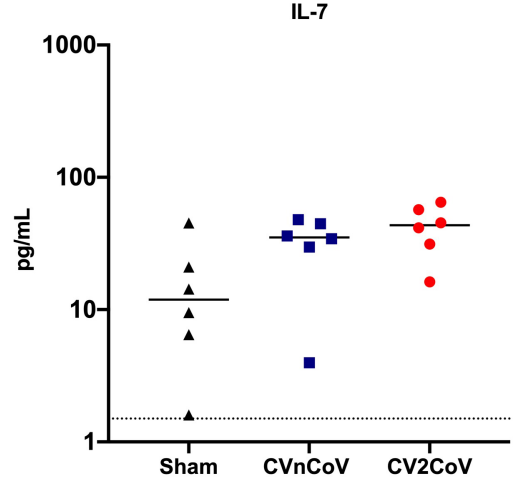

i

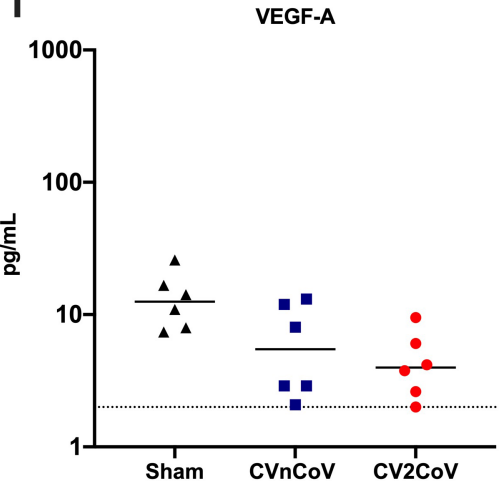

Extended Data Fig. 1 | Innate cytokine induction following mRNA

immunization (6/group). Sera isolated $24 \mathrm{~h}$ post first injection were analyzed for a panel of 19 cytokines associated with viral infection using a U-PLEX Viral Combo kit from Meso Scale Discovery. Changes in cytokine levels above the detection limits were detectable for 9 cytokines. Each dot represents an individual animal, bars depict the median and the dotted line shows limit of detection. Statistical analysis was performed using two-tailed nonparametric Mann-Whitney test. 


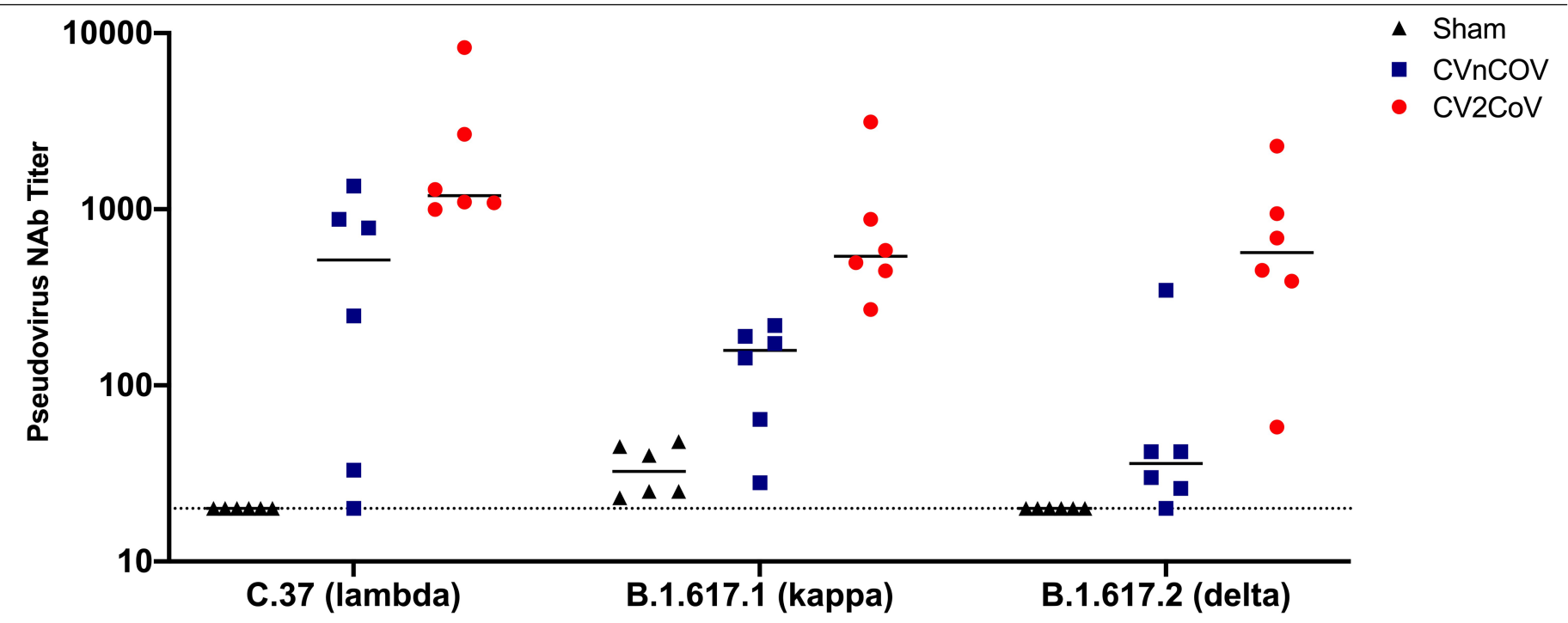

Extended Data Fig. 2 | Neutralizing antibody titers against variants.

against C.37 (Lambda), B.1.617.1 (Kappa) and B.1.617.2 (Delta) variants. Each dot Animals (6/group) were vaccinated twice with $12 \mu \mathrm{g}$ of CVnCoV or CV2CoV on represents an individual animal, bars depict the median and the dotted line $\mathrm{d} 0$ and $\mathrm{d} 28$ or remained untreated as negative controls (sham). Sera isolated on shows limit of detection.

d42 (week 6) were analyzed for pseudovirus neutralizing antibody titers 
a

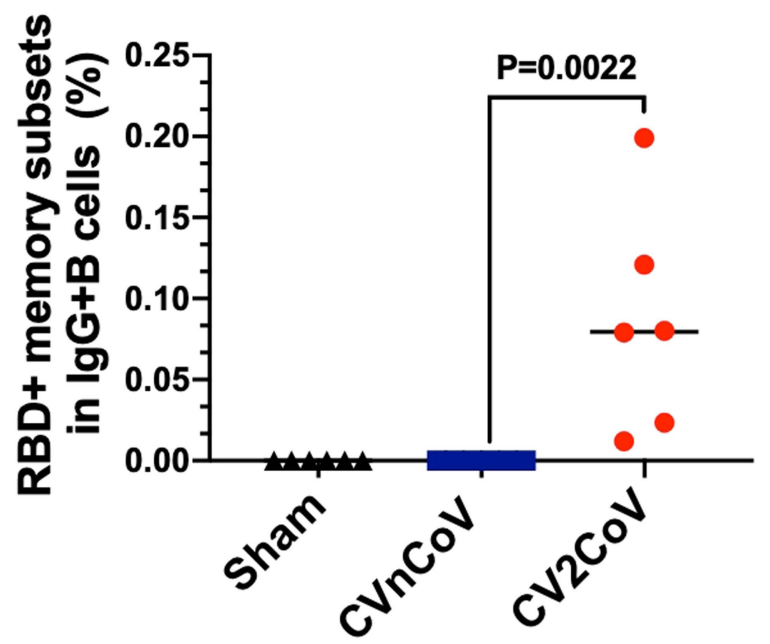

C

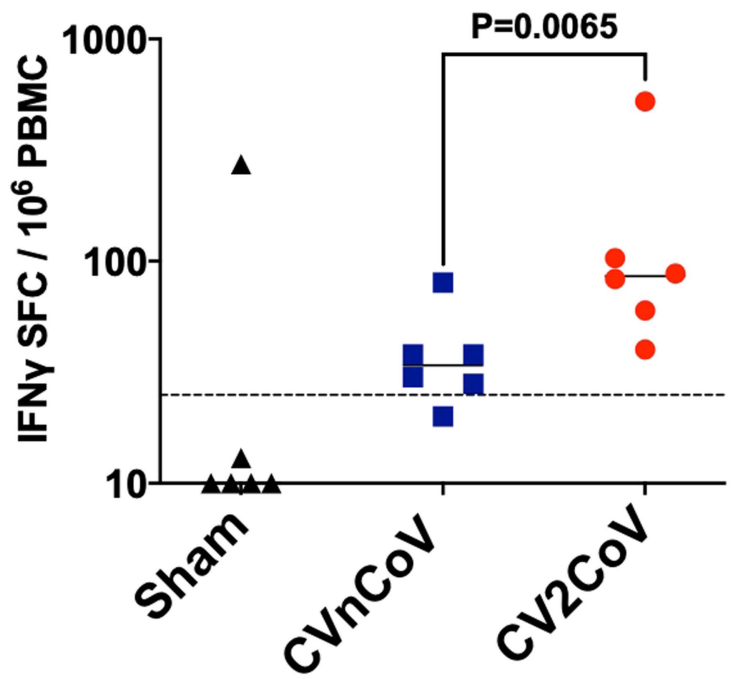

Extended Data Fig. 3 | Memory B and T cell immune responses day 42 following immunization. $\mathrm{PBMCs}$ from negative control (sham), $\mathrm{CVnCoV}$ or $\mathrm{CV} 2 \mathrm{CoV}$ vaccinated animals (6/group) isolated on $\mathrm{d} 42$ of the experiment were stained for (a) RBD and (b) Spike-specific activated memory B cells and analyzed by high-parameter flow cytometry. IFN $\gamma$ responses to pooled spike b
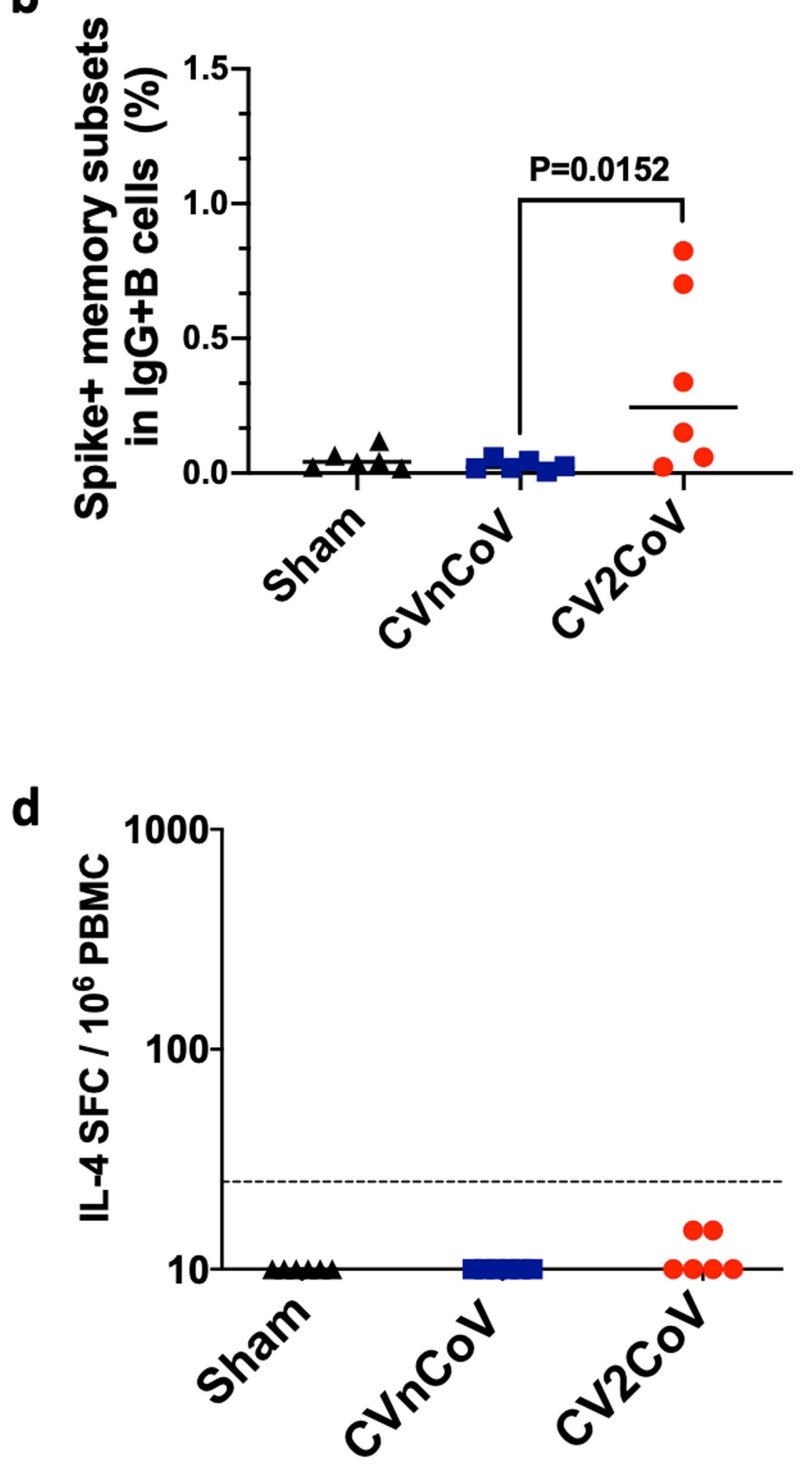

peptides were analyzed via ELISPOT (c). Each dot represents an individual animal, bars depict the median and the dotted line shows limit of detection. Statistical analysis was performed using two-tailed nonparametric

Mann-Whitney test. $\mathrm{PBMC}=$ peripheral blood mononuclear cell; $\mathrm{SFC}=\mathrm{spot}$ forming cells. 
a

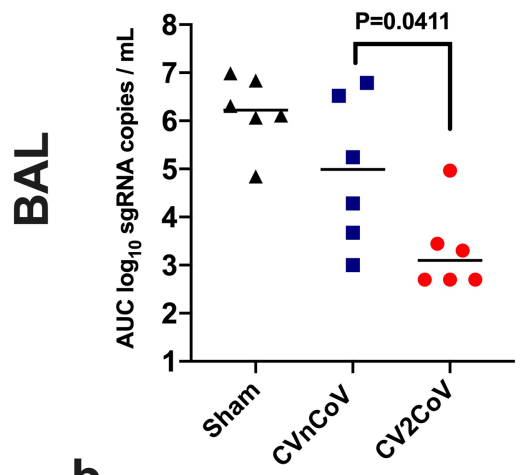

b

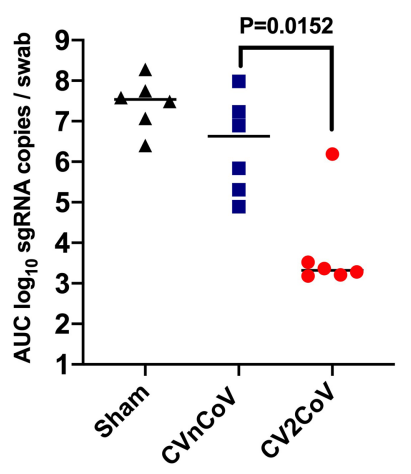

C

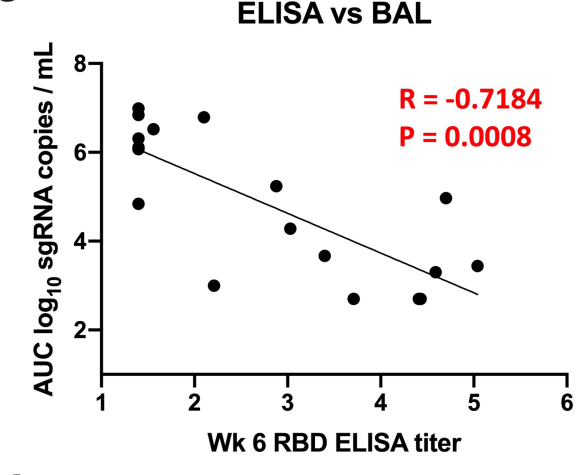

d

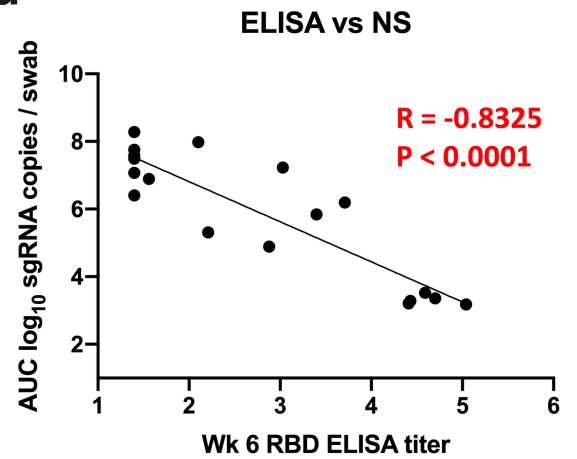

e

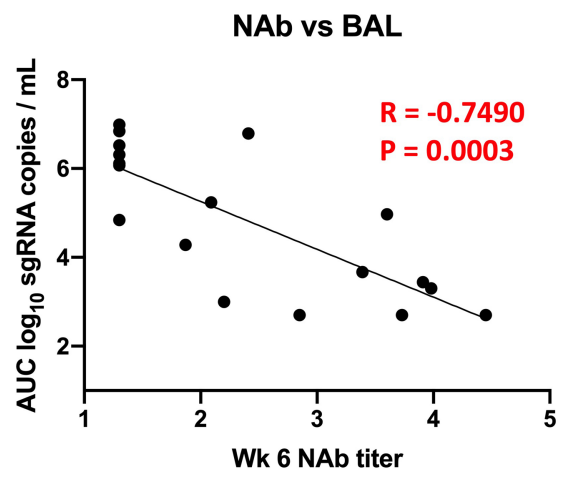

f

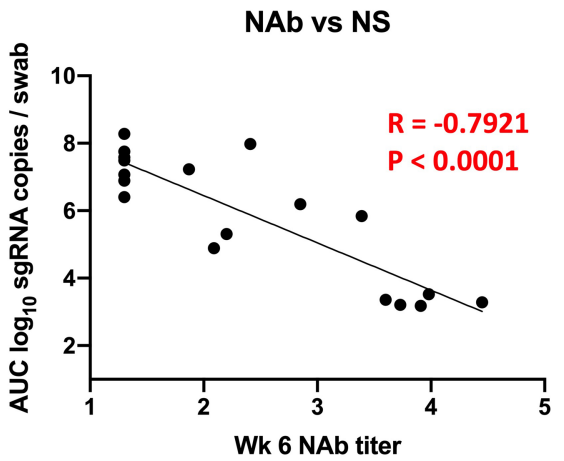

Extended Data Fig. 4 | Binding and neutralizing antibody titers correlate with protection against SARS-CoV-2. Summary of area under curve (AUC) viral load values following SARS-CoV-2 challenge in BAL and nasal swab samples (6/group) (a, b); antibody correlates of protection for binding antibodies (c, d) and neutralizing antibodies (e,f). Statistical analysis was performed using two-tailed nonparametric Mann-Whitney test. Correlations was analyzed by two-sided Spearman rank-correlation test. $\mathrm{NAbs}=$ neutralizing antibodies, $\mathrm{BAL}=$ bronchoalveolar lavage $\mathrm{NS}=$ nasal swab. 


\section{Article}

a

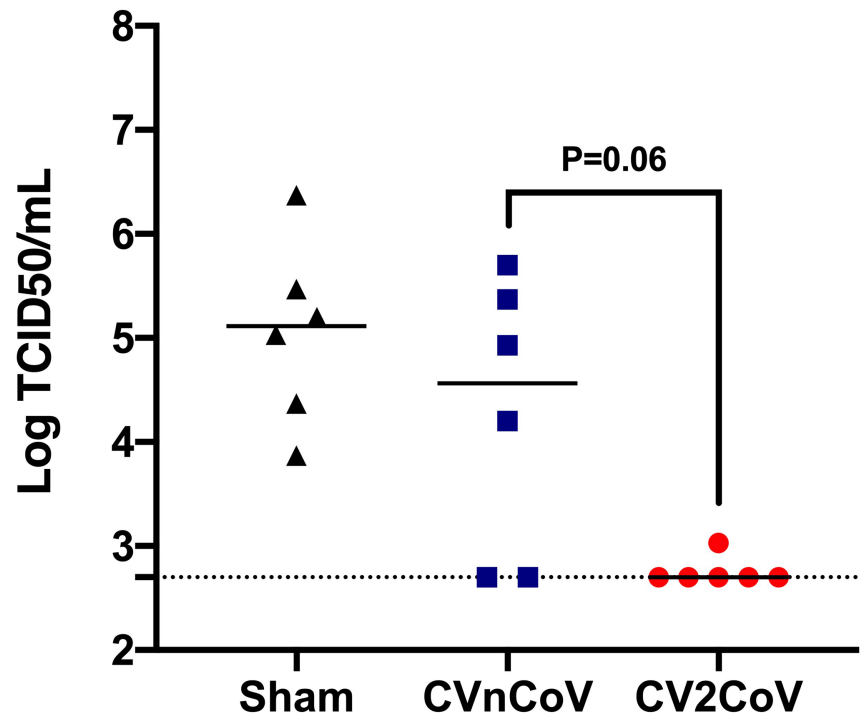

Extended Data Fig. 5 | Infectious virus titers after SARS-CoV-2 challenge (6/group). Infectious virus titers of BAL and nasal swab samples collected 2 days post challenge were analyzed by $\mathrm{TCID}_{50}$ assays. Each dot represents an

\section{b}

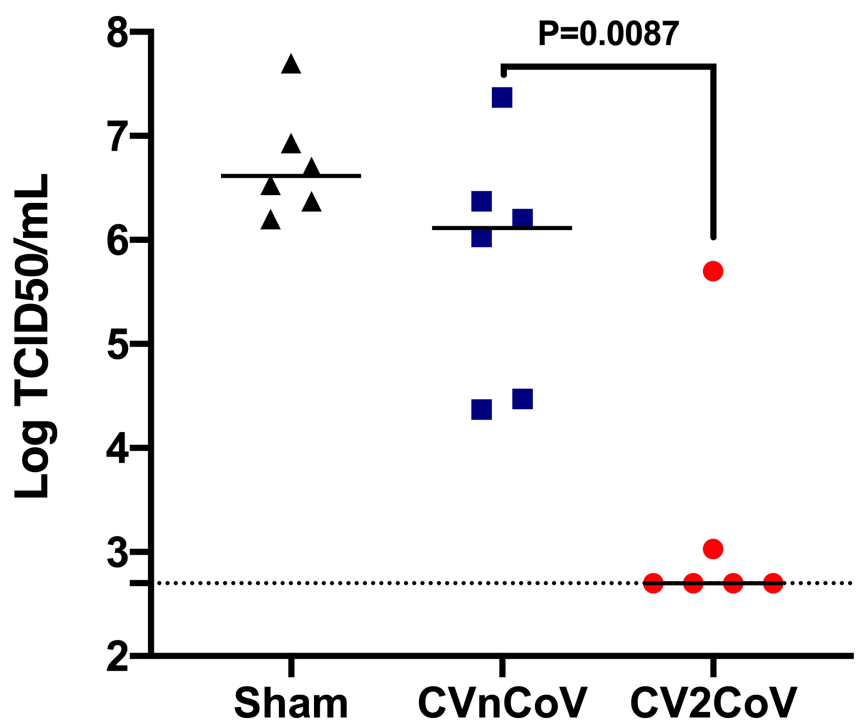

individual animal, bars depict the median and the dotted line shows limit of detection. Statistical analysis was performed using two-tailed nonparametric Mann-Whitney test. 
a

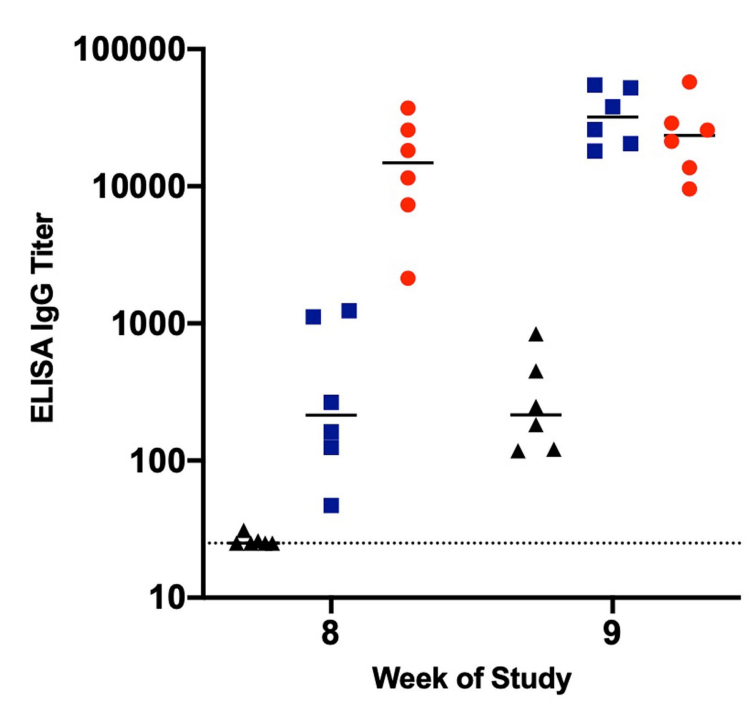

Extended Data Fig. 6 | Post-challenge binding and neutralizing antibody responses (6/group). Negative control (sham) or animals vaccinated on d0 and $\mathrm{d} 28$ of the experiment with $12 \mu \mathrm{g}$ of $\mathrm{CVnCoV}$ or $\mathrm{CV} 2 \mathrm{CoV}$ as indicated were subjected to challenge infection using $1.0 \times 10^{5} \mathrm{TCID}_{50}$ SARS-CoV-2 via intranasal (IN) and intratracheal (IT) routes. (a) Titers of RBD binding antibodies and b

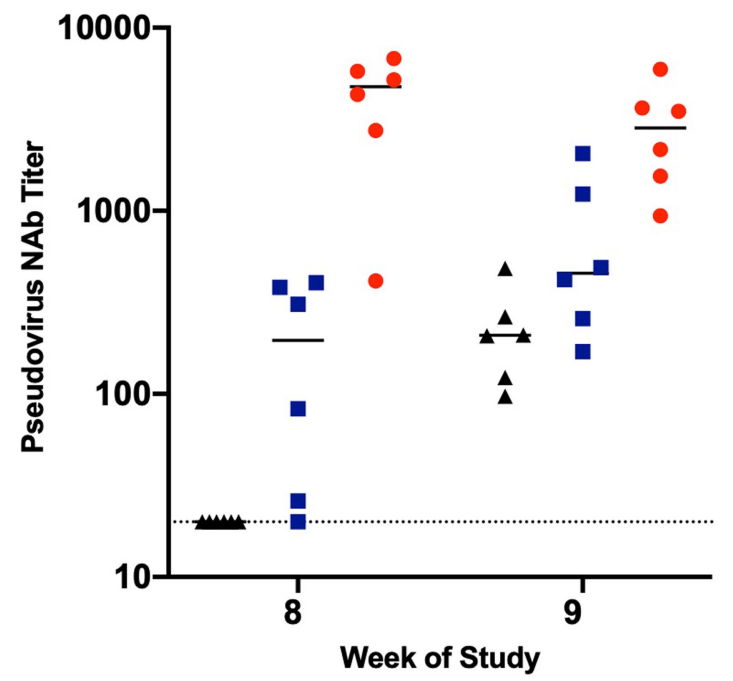

- Sham

- $\mathrm{CVnCOV}$

- $\mathrm{CV} 2 \mathrm{CoV}$

(b) pseudovirus neutralizing antibodies against ancestral SARS-CoV-2 strain were evaluated before (week 8) and a week after challenge infection (week 9). Each dot represents an individual animal, bars depict the median and the dotted line shows limit of detection. Statistical analysis was performed using two-tailed nonparametric Mann-Whitney test. NAbs = neutralizing antibodies. 


\section{Article}

a

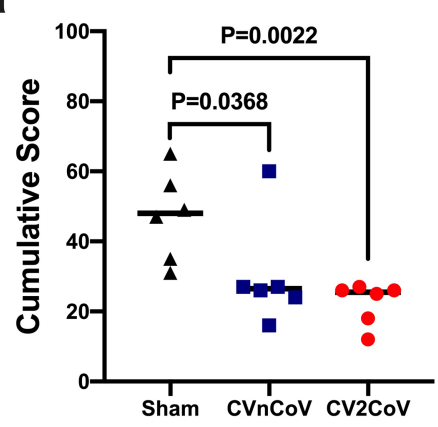

b

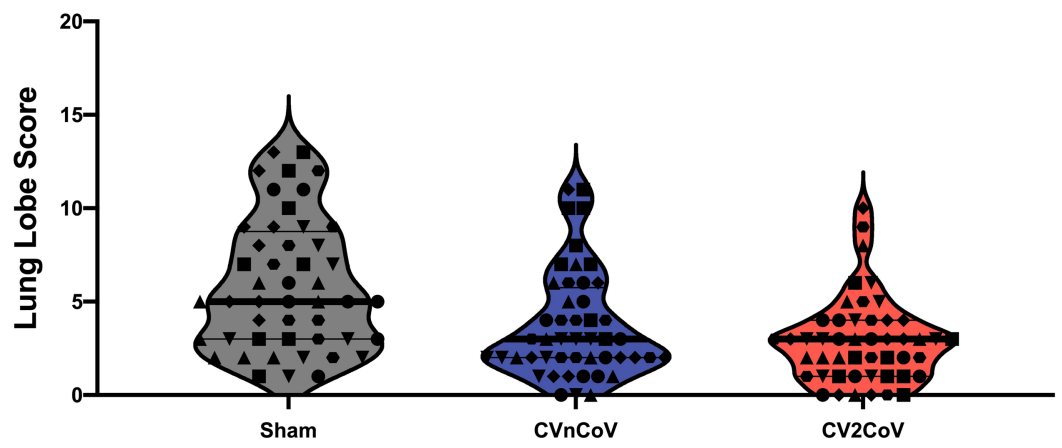



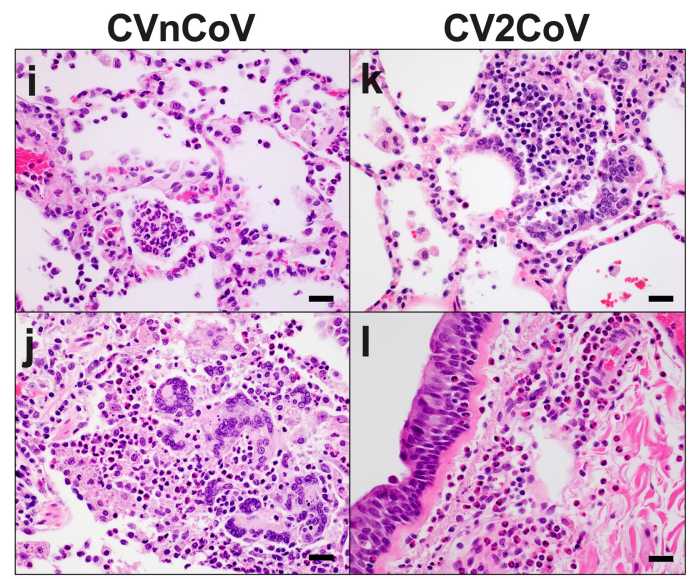

Extended Data Fig. $7 \mid \mathrm{CVnCoV}$ and CV2CoV protect the lungs from pathological changes upon viral challenge (6/group). Eight lung lobes ( 4 sections from right and left, caudal to cranial) were assessed and scored (1-4) for each of the following lesions: 1) Interstitial inflammation and septal thickening 2) Eosinophilic interstitial infiltrate 3) Neutrophilic interstitial infiltrate 4) Hyaline membranes 5) Interstitial fibrosis 6) Alveolar infiltrate, macrophage 7) Alveolar/Bronchoalveolar infiltrate, neutrophils 8) Syncytial cells 9) Type II pneumocyte hyperplasia 10) Broncholar infiltrate, macrophage 11) Broncholar infiltrate, neutrophils 12) BALT hyperplasia 13) Bronchiolar/ peribronchiolar inflammation 14) Perivascular, mononuclear infiltrates 15) Vessels, endothelialitis. Each feature assessed was assigned a score of $0=$ no significant findings; 1 = minimal $; 2$ = mild $; 3=$ moderate $;$ = marked $/$ severe . (a) Cumulative scores per animal (b) Cumulative scores per lung lobe. Individual animals are represented by symbols. Representative histopathology from sham vaccinated $(\mathbf{c} \cdot \mathbf{h}), \mathrm{CnVCoV}$ vaccinated $(\mathbf{i}, \mathbf{j})$, and $\mathrm{Cv} 2 \mathrm{CoV}$ vaccinated (k, I) animals showing (c, d, inset) alveolar macrophage infiltrate, (e, f, inset) syncytial cells (arrowheads) and type II pneumocyte hyperplasia, inset (g, h, inset) bronchiolar epithelial necrosis with neutrophilic infiltrates (i) alveolar neutrophilic infiltrate and alveolar septal thickening $(\mathbf{j})$ focal consolidation with inflammation composed of macrophages, neutrophils, and syncytial cells (k) focal pneumocyte hyperplasia, syncytial cells and inflammatory infiltrates (l) peribronchiolar inflammation. Statistical analysis was performed using two-tailed nonparametric Mann-Whitney test. Scale bars: 100 microns (c), 50 microns (e,g) 20 microns (i-1). BALT bronchus associated lymphoid tissue. 


\section{Reporting Summary}

Nature Portfolio wishes to improve the reproducibility of the work that we publish. This form provides structure for consistency and transparency in reporting. For further information on Nature Portfolio policies, see our Editorial Policies and the Editorial Policy Checklist.

\section{Statistics}

For all statistical analyses, confirm that the following items are present in the figure legend, table legend, main text, or Methods section.

$\mathrm{n} / \mathrm{a} \mid$ Confirmed

$\bigotimes$ The exact sample size $(n)$ for each experimental group/condition, given as a discrete number and unit of measurement

\ A statement on whether measurements were taken from distinct samples or whether the same sample was measured repeatedly

The statistical test(s) used AND whether they are one- or two-sided

Only common tests should be described solely by name; describe more complex techniques in the Methods section.

\ A description of all covariates tested

$\square$ A description of any assumptions or corrections, such as tests of normality and adjustment for multiple comparisons

A full description of the statistical parameters including central tendency (e.g. means) or other basic estimates (e.g. regression coefficient)

AND variation (e.g. standard deviation) or associated estimates of uncertainty (e.g. confidence intervals)

For null hypothesis testing, the test statistic (e.g. $F, t, r$ ) with confidence intervals, effect sizes, degrees of freedom and $P$ value noted

Give $P$ values as exact values whenever suitable.

Х $\square$ For Bayesian analysis, information on the choice of priors and Markov chain Monte Carlo settings

Х $\square$ For hierarchical and complex designs, identification of the appropriate level for tests and full reporting of outcomes

$\square \bigotimes$ Estimates of effect sizes (e.g. Cohen's $d$, Pearson's $r$ ), indicating how they were calculated

\section{Our web collection on statistics for biologists contains articles on many of the points above.}

\section{Software and code}

Policy information about availability of computer code

Data collection Flow Jo Version 10.6.2 was used

Data analysis Virologic and immunologic data analysis was performed using GraphPad Prism 9.0.0 (GraphPad Software). Specifically, cytokine data analysis was done using DISCOVERY WORKBENCH ${ }^{\circledR} 4.0$ software.

For manuscripts utilizing custom algorithms or software that are central to the research but not yet described in published literature, software must be made available to editors and

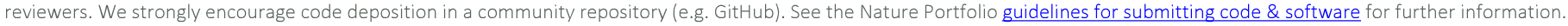

\section{Data}

Policy information about availability of data

All manuscripts must include a data availability statement. This statement should provide the following information, where applicable:

- Accession codes, unique identifiers, or web links for publicly available datasets

- A description of any restrictions on data availability

- For clinical datasets or third party data, please ensure that the statement adheres to our policy 
Please select the one below that is the best fit for your research. If you are not sure, read the appropriate sections before making your selection.

$\bigotimes$ Life sciences $\quad \square$ Behavioural \& social sciences $\quad \square$ Ecological, evolutionary \& environmental sciences

For a reference copy of the document with all sections, see nature.com/documents/nr-reporting-summary-flat.pdf

\section{Life sciences study design}

All studies must disclose on these points even when the disclosure is negative.

\begin{tabular}{l|l} 
Sample size & Sample size includes $\mathrm{N}=18$ animals ( $\mathrm{N}=6$ animals for each vaccine or sham control groups). Based on our previous experience with SARS-CoV-2 \\
in cynomolgus as well as rhesus macaques, this sample size provides sufficient power to determine differences in protective efficacy of both
\end{tabular} vaccinated groups compared with the sham controls.

Data exclusions No data were excluded.

Replication Virologic and immunologic measures were performed in duplicate. Technical replicates were minimally different. Attempts in replication were succesful

Randomization Animals were balanced for age and gender and otherwise randomly allocated to groups. All other sample randomization through out the study was random.

Blinding All immunologic and virologic assays were performed blinded.

\section{Reporting for specific materials, systems and methods}

We require information from authors about some types of materials, experimental systems and methods used in many studies. Here, indicate whether each material, system or method listed is relevant to your study. If you are not sure if a list item applies to your research, read the appropriate section before selecting a response.

\begin{tabular}{|c|c|c|c|}
\hline \multicolumn{2}{|r|}{ Materials \& experimental systems } & \multicolumn{2}{|c|}{ Methods } \\
\hline $\mathrm{n} / \mathrm{a}$ & Involved in the study & $\mathrm{n} / \mathrm{a}$ & Involved in the study \\
\hline & $\bigotimes$ Antibodies & $\bigotimes$ & $\square$ ChIP-seq \\
\hline & Xukaryotic cell lines & Х & $\square$ Flow cytometry \\
\hline 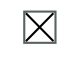 & $\square$ Palaeontology and archaeology & $\bigotimes$ & $\square$ MRI-based neuroimaging \\
\hline & Х Animals and other organisms & & \\
\hline$\bigotimes$ & $\square$ Human research participants & & \\
\hline Х & $\square$ Clinical data & & \\
\hline Х & $\square$ Dual use research of concern & & \\
\hline
\end{tabular}

\section{Antibodies}

Antibodies used

For ELISA assay: anti-macaque IgG HRP (NIH NHP Reagent Program); for ELISPOT assay: mouse anti-human IFN- $\gamma$ monoclonal antibody (BD Pharmingen), IL-4 capture monoclonal antibody (Mabtech), rabbit polyclonal anti-human IFN- $y$ Biotin (U-Cytech) and Streptavidin-alkaline phosphatase antibody ( IFN-y: Southern Biotechnology/IL-4 (Mabtech). For B cell ICS assay: monoclonal antibodies against CD45 (clone D058-1283, BUV805), CD3 (clone SP34.2, APC-Cy7), CD7 (clone M-T701, Alexa700), CD123 (clone 6H6, Alexa700), CD11c (clone 3.9, Alexa700), CD20 (clone 2H7, PE-Cy5), IgA (goat polyclonal antibodies, APC), IgG (clone G18-145, BUV737), IgM (clone G20-127, BUV396), IgD (goat polyclonal antibodies, PE), CD80 (clone L307.4, BV786), CD95 (clone DX2, BV711), CD27 (clone M-T271, BUV563), CD21 (clone B-ly4, BV605), CD14 (clone M5E2, BV570) and CD138 (clone DL-101, PE-CF594).

Validation

mAbs were used according to manufacturer's instructions and previously published methods; mAbs were validated and titrated for specificity prior to use

\section{Eukaryotic cell lines}

Policy information about cell lines

Cell line source(s)

HEK293T and Vero E6 cells Commerically purchased (ATCC)

Authentication

Mycoplasma contamination
Cell lines were not authenticated.

Negative for mycoplasma 


\section{Animals and other organisms}

Policy information about studies involving animals; ARRIVE guidelines recommended for reporting animal research

\begin{tabular}{|c|c|}
\hline Laboratory animals & 18 male and female cynomolgus macaques (Macaca fascicularis), 3-20 years old \\
\hline Wild animals & None \\
\hline Field-collected samples & None \\
\hline Ethics oversight & $\begin{array}{l}\text { All animal studies were conducted in compliance with all relevant local, state, and federal regulations and were approved by the } \\
\text { Bioqual Institutional Animal Care and Use Committee (IACUC). }\end{array}$ \\
\hline
\end{tabular}

Note that full information on the approval of the study protocol must also be provided in the manuscript. 